\title{
OTELO SURVEY: DEEP BVRI BROADBAND PHOTOMETRY OF THE GROTH STRIP. II. OPTICAL PROPERTIES OF X-RAY EMITTERS
}

\author{
M. Pović ${ }^{1}$, M. Sánchez-Portal ${ }^{2}$, A. M. Pérez García ${ }^{1,8}$, A. Bongiovanni ${ }^{1,8}$, J. CepaA ${ }^{3,9}$, E. Alfaro ${ }^{4}$, H. Castañeda ${ }^{1,8,10}$, \\ M. Fernández Lorenzo ${ }^{1}$, J. Gallego ${ }^{5}$, J. I. González-Serrano ${ }^{6}$, J. J. González ${ }^{7}$, and M. A. Lara-López ${ }^{1}$ \\ ${ }^{1}$ Instituto de Astrofísica de Canarias, La Laguna, Spain; mpovic@iac.es \\ ${ }^{2}$ Herschel Science Centre, ESAC/INSA, P.O. Box 78, 28691 Villanueva de la Cañada, Madrid, Spain; miguel.sanchez@sciops.esa.int \\ ${ }^{3}$ Departamento de Astrofísica, Universidad de La Laguna, La Laguna, Spain \\ ${ }^{4}$ Instituto de Astrofísica de Andalucía-CSIC, Granada, Spain \\ ${ }^{5}$ Departamento de Astrofísica y CC. de la Atmósfera, Universidad Complutense de Madrid, Madrid, Spain \\ ${ }^{6}$ Instituto de Física de Cantabria, CSIC-Universidad de Cantabria, Santander, Spain \\ ${ }^{7}$ Instituto de Astronomía UNAM, México D.F, México \\ Received 2008 October 20; accepted 2009 October 14; published 2009 November 5
}

\begin{abstract}
The Groth field is one of the sky regions that will be targeted by the OSIRIS Tunable Filter Emission Line Object survey in the optical $820 \mathrm{~nm}$ and $920 \mathrm{~nm}$ atmospheric windows. In the present paper, public Chandra X-ray data with total exposure time of $200 \mathrm{ks}$ are analyzed and combined with optical broadband data of the Groth field, in order to study a set of optical structural parameters of the X-ray emitters and its relation with X-ray properties. To this aim, we processed the raw, public X-ray data using the Chandra Interactive Analysis of Observations, and determined and analyzed different structural parameters, in order to produce a morphological classification of X-ray sources. We present the morphology of $340 \mathrm{X}$-ray emitters with optical counterpart detected. Objects have been classified by X-ray type using a diagnostic diagram relating X-ray-tooptical ratio $(\mathrm{X} / \mathrm{O})$ to hardness ratio. We did not find any clear correlation between X-ray and morphological types. We analyzed the angular clustering of X-ray sources with optical counterpart using two-point correlation functions. A significant positive angular clustering was obtained from a preliminary analysis of four subsamples of the X-ray sources catalog. The clustering signal of the optically extended counterparts is similar to that of strongly clustered populations like red and very red galaxies, suggesting that the environment plays an important role in active galactic nuclei phenomena. Finally, we combined optical structural parameters with other X-ray and optical properties, and we confirmed an anticorrelation between the $\mathrm{X} / \mathrm{O}$ ratio and the Abraham concentration index, which might suggest that early-type galaxies have lower Eddington rates than those of late-type galaxies.
\end{abstract}

Key words: galaxies: active - X-rays: galaxies

Online-only material: color figures, machine-readable table

\section{INTRODUCTION}

Optical broadband data combined with X-ray data provide a powerful tool to study active galactic nuclei (AGNs) properties (Georgakakis et al. 2006; Steffen et al. 2006; Barcons et al. 2007). Also, optical imaging surveys allow a morphological classification of all sufficiently resolved sources. This is essential to reveal the nature of AGN host galaxies (e.g., Fiore et al. 2001; Hasan 2007), although the morphological classification becomes difficult at high redshift, due to the reduced resolution of images. Moreover, several works (Ferrarese \& Merritt 2000; Gebhardt et al. 2000; Graham et al. 2001) have shown that the AGNs are directly related with some host galaxy properties, particularly with their bulges. Kauffmann et al. (2003) have pointed out that the AGNs in the local universe are hosted predominantly in bulge-dominated galaxies. More recently, Hasan (2007) using Hubble Space Telescope (HST) and Chandra data of the GOODS South field found that the most moderate luminosity AGNs hosts in the redshift range $z \sim 0.4-1.3$ are bulge-dominated. However, Gabor et al. (2009) found that X-ray selected AGN host

\footnotetext{
8 Also at Departamento de Astrofísica, Universidad de La Laguna, La Laguna, Spain.

9 Also at Instituto de Astrofísica de Canarias, La Laguna, Spain.

${ }^{10}$ Also at Departamento de Física, Escuela Superior de Física y, Matemática, IPN, México D.F., México.
}

galaxy morphologies at redshifts $0.3<z<1.0$ span a substantial range of morphological types between those of earlytype (bulge-dominated) and late-type (disc-dominated) systems. Finally, a tight correlation between black hole mass and the bulge dispersion velocity has been well established (Ferrarese \& Merritt 2000; Gebhardt et al. 2000), as well as the black hole mass correlation with the optical concentration of bulges (Graham et al. 2001).

While the analysis of optical data provides valuable information on the host galaxy, deep X-ray extragalactic surveys allow for an extremely efficient detection of the population of AGNs, to investigate their time evolution, and to shed light on their triggering mechanisms. This exceptional effectiveness finding AGNs arises largely because X-ray selection has reduced absorption bias, and minimal dilution by host galaxy starlight, thus allowing efficient optical spectroscopic follow-ups of high probability AGN candidates with faint optical counterparts. The Chandra and XMM-Newton observatories have revolutionized this field of research, generating the most sensitive X-ray surveys ever performed (Giacconi et al. 2002; Alexander et al. 2003). In fact, deep surveys produced by these observatories have increased the resolved fraction of the cosmic X-ray background (CXRB) to about $90 \%$ in the $0.5-2 \mathrm{keV}$ range (Moretti et al. 2003; Bauer et al. 2004; Worsley et al. 2004). A large part of the detected X-ray sources are AGN $(\geqslant 70 \%)$, although 
X-ray emitters include a mix of different types of objects such as galaxy clusters and stars. Moreover, these powerful observatories make it possible to access the hard $(2-10 \mathrm{keV})$ range, allowing to directly probe the AGN activity not contaminated by star formation processes. These hard X-ray surveys are then capable to detect all but the most absorbed (Compton thick) sources. Therefore, they provide the most complete and unbiased samples of AGNs (Mushotzky 2004; Brandt \& Hasinger 2005). Number counts relations have now been determined (e.g., Cappelluti et al. 2007), tightly constrained over a wide range of fluxes, although with some evidence for field-to-field variations. Such variations are expected at some level owing to "cosmic statistics" associated with large-scale structures and clustering features that have been detected in the X-ray sky (Barger et al. 2003; Yang et al. 2003; Gilli et al. 2003, 2005).

The OTELO (OSIRIS Tunable Filter Emission Line Object) project (Cepa et al. 2005, 2008) is an emission-line objects survey, using the OSIRIS (Optical System for Imaging and low Resolution Integrated Spectroscopy) tunable filters at the GTC $10.4 \mathrm{~m}$ telescope. OTELO will explore the wavelength intervals through the $\mathrm{OH}$ emission line forest, centered at 815 and $920 \mathrm{~nm}$, with spectral widths of 15 and $18 \mathrm{~nm}$, respectively. OTELO is a long-term survey based on a new approach, since the selection of objects will be done by their emission-line flux. Therefore, it will introduce a new parameter in the largescale survey space. OTELO will be unique, and the first largescale emission-line survey fully exploiting the sensitivity and spectral resolution (for deblending [N II] from $\mathrm{H} \alpha$ ) of tunable filters. The OTELO survey, more than 1 mag deeper (with $\mathrm{H} \alpha$ equivalent widths $\geqslant 2$ for a depth of $10^{-18} \mathrm{erg} \mathrm{cm}^{-2} \mathrm{~s}^{-1}$ at $5 \sigma$ ) than other emission line surveys, will provide a wide variety of astronomical sources: star-forming galaxies, starburst galaxies, emission-line ellipticals, AGNs, QSOs, Ly $\alpha$ emitters, peculiar stars, etc. With these data, several important scientific problems will be addressed, including star formation rate (SFR) and metallicity evolution of galaxies, and AGN and QSO evolution. Deep imaging programs as well as several VLT surveys for GOODS (Dickinson et al. 2004), COSMOS (Capak et al. 2007; Scoville et al. 2007; Lilly et al. 2007), and SXDS (Furusawa et al. 2008) fields will serve as preparatory work for OTELO. Likewise, we have performed a deep BVRI broadband survey (Cepa et al. 2008) in one of the fields of the Groth strip selected for the OTELO survey. Here we combine these optical data with X-ray information, in order to tackle the study of the AGN population, one of the objectives of the OTELO project. The results presented here will complement the analysis of tunable filter data.

We present the analysis of public, deep (200 ks) Chandra/ ACIS observations of three fields comprising the original Groth-Westphal strip (GWS), gathered from the Chandra Data Archive, and combined with optical BVRI data from our broadband survey carried out with the $4.2 \mathrm{~m}$ William-Herschel Telescope (WHT) at La Palma (see Cepa et al. 2008, for a detailed description). We have performed a detailed morphological classification of a large sample of X-ray emitters with optical counterparts in the GWS field; a previous work (Pierce et al. 2007) presented a morphological analysis of the optical counterparts of X-ray emitters in the GWS, though their sample was smaller, about one-third of the one presented in this paper. Moreover, we present a catalog containing, for each X-ray object, measured structural parameters and its morphological type. We have implemented an innovative approach to the optical study of $\mathrm{X}$-ray emitters by investigating correlations between broadband optical and X-ray parameters, specifically the X-ray-to-optical ratio $(\mathrm{X} / \mathrm{O})$ and hardness ratios (HRs), and optical structural parameters that provide information about the host galaxy morphology and populations, like asymmetry, concentration index, and optical colors. Finally, we analyze the clustering properties of our sample of X-ray detected AGNs.

The paper is structured as follows: in Section 2, we describe the observational data, including X-ray data processing and source detection, as well as optical broadband data and the selection of the sample of X-ray sources with optical counterparts. Section 3 reviews the procedures for computing optical structural parameters, as well as the morphological analyses carried out. The reliability of different structural parameters used in the morphological classification is also discussed. Section 4 presents the X-ray type classification, and the relationship between X-ray and optical structural properties. The catalog presenting the morphological classification of X-ray emitters with optical counterparts and their structural parameters is presented in the electronic edition. A complete X-ray public catalog can be found in the All-wavelength Extended Groth strip International Survey (AEGIS; Laird et al. 2009).

\section{OBSERVATIONAL DATA}

\subsection{X-ray Data}

\subsubsection{Observations and Data Processing}

Three contiguous fields centered at the original HST GWS have been observed by the AEGIS collaboration, using the Chandra/ACIS-I instrument. All data sets have been gathered from the Chandra Data Archive (http://asc.harvard.edu/ $\mathrm{cda} /$ ) using the Chaser tool. Total exposure time in each field is about $200 \mathrm{ks}$. The total field size of ACIS-I chips 0, 1, 2, and 3 is $16.9 \times 16.9$ (ACIS-S chips 2 and 3 were also used, but their field of view (FOVs) do not overlap with our optical data and therefore were not used). The FAINT and VFAINT telemetry modes have been used, with telemetry saturation rates of 170 and 67 events/s, respectively. Due to the lack of bright sources within the fields, we assume that there are no pile-up effects in any observation. Table 1 summarizes the main characteristics of the Chandra observations.

We processed the data using the Chandra Interactive Analysis of Observations (CIAO; http://cxc.harvard.edu/ciao/), v4.0 and Calibration Data Base (CALDB) v3.4.0. Standard reduction procedures have been applied, as described in the CIAO Science Threads to produce new level 2 event files. The output level 2 event files have been restricted to the $0.5-8 \mathrm{keV}$ range to avoid high background spectral regions. Furthermore, we analyzed the source-free light curves in order to define additional good time intervals (GTI). To this end, we applied the CIAO detection program celldetect with a threshold $\mathrm{S} / \mathrm{N}=3$. The detected sources have been removed upon creation of the time-binned light curves, that have been then used for the computation of the GTIs. A $\pm 3 \sigma$ rejection criterion has been applied to remove high background intervals. After filtering for the additional GTIs, the level 2 event files belonging to the same target were co-added. To this end, we first improved the absolute astrometry (the overall $90 \%$ uncertainty circle of Chandra X-ray absolute position has a radius of 0.'6), applying corrections for translation, scale, and rotation to the world coordinate system (WCS) of each data file, by comparing two sets of source lists of the same sky region. Average positional accuracy improvement is $6.3 \%$ with respect to the original 
Table 1

Chandra Observations Log for the Three Pointings of the GWS Field

\begin{tabular}{|c|c|c|c|c|c|}
\hline $\begin{array}{l}\text { Obs. } \\
(1)\end{array}$ & $\begin{array}{c}\text { R.A. } \\
\text { (2) }\end{array}$ & $\begin{array}{c}\text { Decl. } \\
(3)\end{array}$ & $\begin{array}{c}\text { SDP } \\
(4) \\
\end{array}$ & $\begin{array}{c}\text { Mode } \\
(5)\end{array}$ & $\begin{array}{c}\text { GTI } \\
(6) \\
\end{array}$ \\
\hline 5853 & $14: 15: 22.5$ & $+52: 08: 26.4$ & 7.6.7.1 & VFAINT & 42.4 \\
\hline 5854 & & & 7.6.7.1 & VFAINT & 50.2 \\
\hline 6222 & & & 7.6.7.1 & VFAINT & 35.2 \\
\hline 6223 & & & 7.6.7.1 & VFAINT & 49 \\
\hline 6366 & & & 7.6.7.1 & VFAINT & 15.96 \\
\hline 7187 & & & 7.6.7.1 & VFAINT & 8 \\
\hline 5851 & $14: 16: 24.5$ & $+52: 20: 02.59$ & 7.6 .7 .1 & VFAINT & 36 \\
\hline 5852 & & & 7.6 .4 & VFAINT & 11.95 \\
\hline 6220 & & & 7.6.7.1 & VFAINT & 36 \\
\hline 6221 & & & 7.6.7.1 & VFAINT & 3.8 \\
\hline 6391 & & & 7.6.7.1 & VFAINT & 9.4 \\
\hline 7169 & & & 7.6.4.1 & VFAINT & 18.2 \\
\hline 7181 & & & 7.6.7.1 & VFAINT & 8 \\
\hline 7188 & & & 7.6.4.1 & VFAINT & 4.2 \\
\hline 7236 & & & 7.6 .4 & VFAINT & 18.8 \\
\hline 7237 & & & 7.6.4.1 & VFAINT & 17.2 \\
\hline 7238 & & & 7.6 .4 & VFAINT & 9.6 \\
\hline 7239 & & & 7.6.4.1 & VFAINT & 16.2 \\
\hline 3305 & $14: 17: 43.0$ & $+52: 28: 25.2$ & 6.8 .0 & FAINT & 27.6 \\
\hline 4357 & & & 6.9 .0 & FAINT & 79.8 \\
\hline 4365 & & & 6.9 .0 & FAINT & 58.2 \\
\hline
\end{tabular}

Notes. Column (1): observation number; Columns (2 and 3): R.A. and Decl. (J2000); Column (4): Standard Data Processing (SDP) version; Column (5): observation format in the Time exposure mode; Column (6): net exposure time (sum of good time intervals, GTI; ks).

astrometry provided by the spacecraft attitude files. Once we have improved the astrometry, the event files have been merged.

The output merged files have been filtered to create event files for several energy bands: full $(0.5-7 \mathrm{keV})$, soft $(0.5-2 \mathrm{keV})$, hard (2-7 keV), hard2 (2-4.5 keV), and vhard (4-7 keV). Unbinned effective exposure maps at a single energy, representative of each band, were created at $2.5 \mathrm{keV}$ (full), $1 \mathrm{keV}$ (soft), $4 \mathrm{keV}$ (hard), $3 \mathrm{keV}$ (hard2), and $5.5 \mathrm{keV}$ (vhard). Resulting maps have units of time $\times$ effective area, i.e., $\mathrm{s} \mathrm{cm}^{2}$ counts photon ${ }^{-1}$.

\subsubsection{Source Detection}

We applied the CIAO wavdetect Mexican-Hat wavelet source detection ${ }^{11}$ program to all bands (full, soft, hard, hard2, and vhard) for the three fields. Several wavelet scales have been applied: $1, \sqrt{2}, 2,2 \sqrt{2}, 4,4 \sqrt{2}, 8,8 \sqrt{2}$, and 16 pixels. Smaller scales are well suited to the detection of small sources, while larger scales are appropriate for more extended sources. Due to the "blank field" nature of the GWS, we do not expect to detect sources at a scale larger than 16 pixels $\left(\sim 8^{\prime \prime}\right)$. For each applied scale, wtransform produces a complete set of detections. We set a significance threshold of $2 \times 10^{-7}$. Since this wavdetect input parameter measures the number of spurious events per pixel, we expect $\approx 0.2$ fake detections per detector of $1024 \times 1024$ pixels, i.e., about 12 spurious sources in the entire catalog ( 4 detectors $\times 5$ bands $\times 3$ fields). The output wavdetect detection files are FITs tables containing the position of sources (R.A. and decl.), count rates in photons $\mathrm{s}^{-1} \mathrm{~cm}^{-2}$, and ancillary information. The final X-ray source catalog has been obtained by cross-matching the output source catalogs

\footnotetext{
11 The fact that the point-spread function (PSF) of X-ray detectors often has Gaussian-like shape motivates our use of the Marr wavelet, or "Mexican Hat" function.
}

in all bands in order to increase the reliability of the detection process. Best match is given by a minimum separation of sources along a great circle. We set a maximum search distance of $2^{\prime \prime}$ (see Section 2.4). We computed the hardness ratios as follows:

$$
\operatorname{HR}\left(\Delta_{1} E, \Delta_{2} E\right)=\frac{\mathrm{CR}\left(\Delta_{1} E\right)-\mathrm{CR}\left(\Delta_{2} E\right)}{\operatorname{CR}\left(\Delta_{1} E\right)+\operatorname{CR}\left(\Delta_{2} E\right)},
$$

where $\Delta_{1} E$ and $\Delta_{2} E$ are different energy bands and $\operatorname{CR}\left(\Delta_{n} E\right)$ is the count rate in a given energy band. Four hardness ratios have been set in this way: $\mathrm{HR}_{1} \equiv \mathrm{HR}$ (hard, soft), $\mathrm{HR}_{1}^{\prime} \equiv \mathrm{HR}$ (hard2, soft), $\mathrm{HR}_{2} \equiv \mathrm{HR}$ (very hard, hard), and $\mathrm{HR}_{2}^{\prime} \equiv \mathrm{HR}$ (very hard, hard2). We also computed the energetic fluxes from count rates. To this end, the mean photon energies in each band have been calculated assuming a power-law spectrum with $\Gamma=1.5$, redshift $z=0.5$, galactic absorption $n_{H}=1.3 \times 10^{20} \mathrm{~cm}^{-2}$, and no intrinsic absorption. We applied the absorption coefficients provided by the TRANNM function within the package PIMMS v3.9b (Mukai 1993). The output catalog has 639 unique X-ray emitters (after removing 20 common sources in the field overlapping area): $67 \%$ (429 sources) have been detected above the $3 \sigma$ significance level, $21 \%$ (134 sources) below $3 \sigma$ level, while 76 objects are located outside the field covered by optical data.

The number of sources detected in each band are 490 (full), 465 (soft), 284 (hard), 266 (hard2), and 121 (vhard), with median fluxes of $1.81 \times 10^{-15}, 5.57 \times 10^{-16}, 2.34 \times 10^{-15}$, $1.58 \times 10^{-15}$, and $3.75 \times 10^{-15} \mathrm{erg} \mathrm{cm}^{-2} \mathrm{~s}^{-1}$, and with limiting fluxes at $3 \sigma$ level of $4.8 \times 10^{-16}, 1.1 \times 10^{-16}, 2.8 \times 10^{-16}$, $1.3 \times 10^{-16}$, and $7.3 \times 10^{-16} \mathrm{erg} \mathrm{cm}^{-2} \mathrm{~s}^{-1}$, respectively. Median errors in fluxes (for objects above the $3 \sigma$ significance level) are $8 \%$ in full band, $10 \%$ in soft band, $12 \%$ in hard band, $14 \%$ in hard 2 band, and $22 \%$ in vhard band. There are 16 sources detected only in hard band, 18 in hard 2 band, and only 3 are observed in vhard band. We find an overabundance of soft X-ray sources in our final catalog. There are only 103 sources detected in this band. This effect can be at least partially explained considering the decrease of telescope + ACIS effective area from some $600 \mathrm{~cm}^{2}$ in the $1-2 \mathrm{keV}$ range (including the $0.5-$ $2 \mathrm{keV}$ band defined as soft), until approximately $200 \mathrm{~cm}^{2}$ at $\sim 6 \mathrm{keV}$. On the other hand, the total background between 0.5 and $7 \mathrm{keV}$ is around four times higher than in the $0.5-2 \mathrm{keV}$ range. The combination of these two factors justifies the high Chandra/ACIS source detection efficiency in the soft X-ray band and the mentioned overabundance. The first version of this catalog was presented in Sánchez-Portal et al. (2007).

We have computed the cumulative number count distributions $(\log N-\log S)$ per square degree in the soft band and compared it with other surveys in order to check the reliability of our source detections. We have chosen the soft band to be able to compare our distribution with that computed by Nandra et al. (2005), and those provided by other deep X-ray surveys, namely Chandra Deep Field South (CDFS; Giacconi et al. 2002), Chandra Deep Field North (CDFN; Alexander et al. 2003), ELAISN1 and ELAIS-N2 (Manners et al. 2003). All distributions are represented in Figure 1. For our sample two curves have been computed, one for the complete sample of X-ray detections and the other only for the X-ray detections that have optical counterparts. The median flux levels are $1.81 \times 10^{-15}$ and $1.97 \times 10^{-15} \mathrm{erg} \mathrm{cm}^{-2} \mathrm{~s}^{-1}$, respectively. For objects brighter than $\sim 10^{-14} \mathrm{erg} \mathrm{s}^{-1} \mathrm{~cm}^{-2}$ both distributions coincide, while for objects fainter than $\sim 10^{-14} \mathrm{erg} \mathrm{s}^{-1} \mathrm{~cm}^{-2}$ the density of X-ray sources with optical counterparts starts to decrease, since the optical sample is incomplete at the depth of our X-ray data. In 


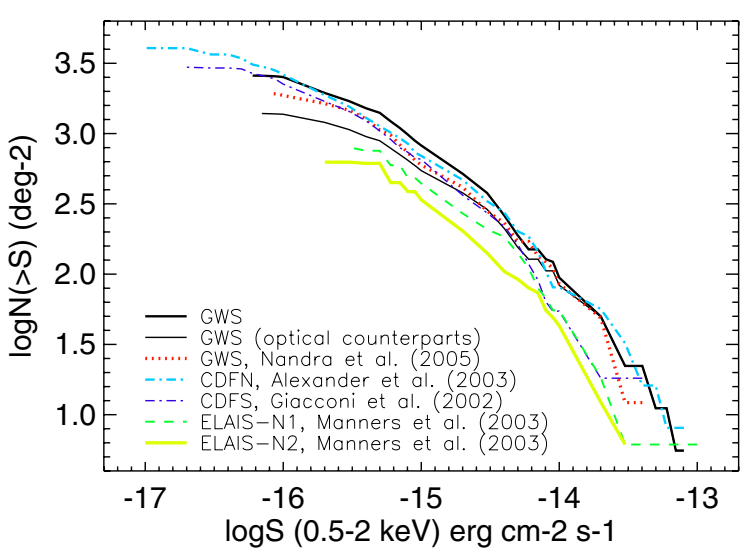

Figure 1. Cumulative $\log N-\log S$ functions for the Groth field in the soft band, for all detected X-ray sources (thick solid line) and for X-ray sources with optical counterpart (thin solid line). Five distributions, with different exposure times and effective areas, have also been represented as comparison: GWS by Nandra et al. (2005; dotted line), CDFN (thick dash-dot-dash line), CDFS (thin dash-dot-dash line), ELAIS-N1 (dash line), and ELAIS-N2 (dash-dot-dot-dash line).

(A color version of this figure is available in the online journal.)

general, at our flux limit, the number counts of our complete X-ray sample are in good agreement with Nandra et al. (2005) and other surveys. We have detected a higher density of sources with flux below $\sim 5 \times 10^{-15} \mathrm{erg} \mathrm{cm}^{-2} \mathrm{~s}^{-1}$, most likely due to the use of different detection algorithms.

\subsection{Comparison with AEGIS Data}

The AEGIS collaboration has recently made public their data sets, including a catalog of X-ray sources that comprises the observations used within this paper. The complete AEGIS X-ray catalog (Laird et al. 2009) is available for public use through the AEGIS web page. ${ }^{12}$ The catalog consists of 1325 sources; from these, 471 are located within the area covered by our data (fields EGS-6, 7, and 8 according to their nomenclature). We have performed a comparison by cross-matching the AEGIS $\mathrm{X}$-ray catalog with our X-ray and broadband optical catalogs. From our 429 sources detected above $3 \sigma$ significance level, 416 coincide with AEGIS sources using a search radius of 2" (388 sources if only unique matches are considered). We found a good agreement between the source counts used in this work and those provided by the AEGIS team: median relative deviations of our data with respect to AEGIS are 2\% (full band), $0.7 \%$ (soft), $5.5 \%$ (hard), and $7.8 \%$ (vhard). Moreover, we have derived X/O flux ratios for our sample and the AEGIS one (in both cases using our optical data), finding a very good agreement in the four common energy bands (full, soft, hard, and ultrahard), as represented in Figure 2.

\subsection{Optical Data}

We observed three pointings in the direction of the GWS, each of them using the $B, V, R$, and $I$ broadband filters. The total area covered is $0.18 \mathrm{deg}^{2}$. The observations have been carried out using the Prime Focus Imaging Platform (PFIP) at the $4.2 \mathrm{~m}$ WHT in the Roque de los Muchachos Observatory (La Palma, Canary Islands). This camera has two CCD detectors $(2 \mathrm{k} \times 4 \mathrm{k})$, with a total field of view of $16^{\prime} \times 16^{\prime}$ and a pixel size of $0 ! 237$. Several exposures have been performed at each pointing, of $600,800,900$, or $1000 \mathrm{~s}$, depending on the filter,

12 http://aegis.ucolick.org/
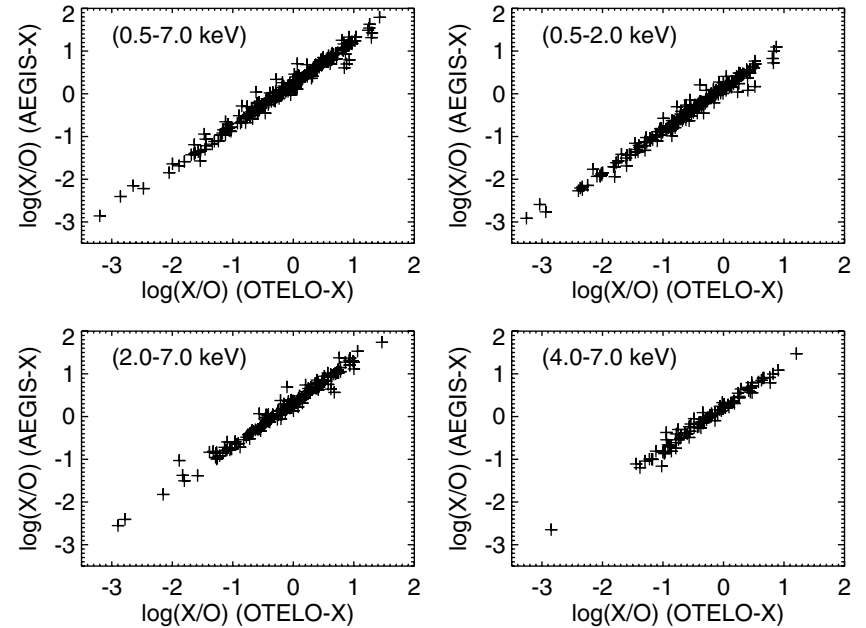

Figure 2. Comparison of $X / O$ flux ratios, obtained by Laird et al. (2009) $\mathrm{X}$-ray data and our optical broadband and X-ray data, in four AEGIS X-ray energy bands (full, soft, hard, and vhard). We found a very good correspondence between our X-ray catalog and the AEGIS one.

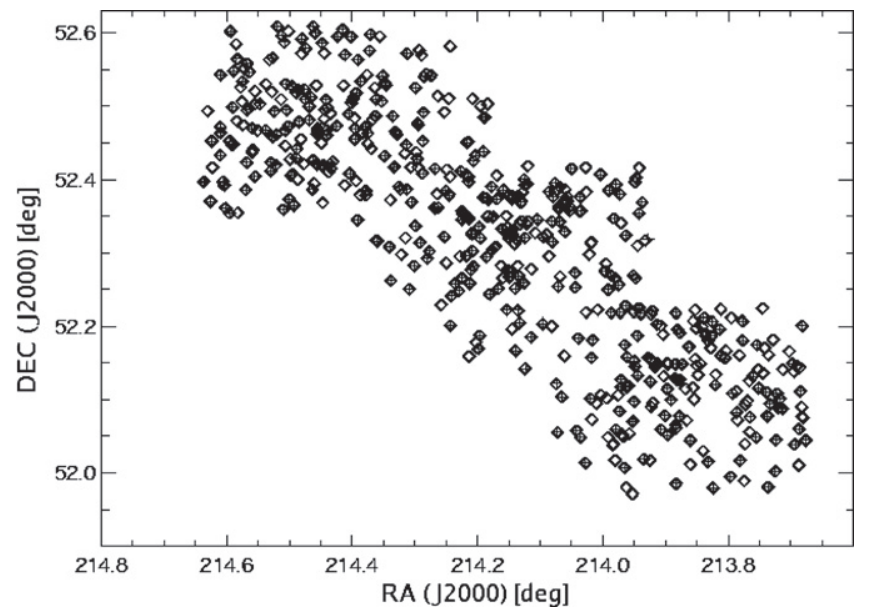

Figure 3. X-ray sources (diamonds) and their optical counterparts (filled diamonds) from the cross-match of X-ray and optical catalogs.

with a dithering of $15^{\prime \prime}$ between consecutive exposures in order to allow eliminating cosmic rays and to fill the gap between detectors. Standard reduction procedures have been applied. Resulting limiting Vega magnitudes are 25, 25, 24.5, and 23.5 in $B, V, R$, and $I$ band, respectively. A detailed description of data reduction and optical source detection can be found in Cepa et al. (2008). The optical catalog used in this work contains data for $\sim 44,000$ objects.

\subsection{The Catalog of Optical Counterparts}

In order to build a sample of X-ray emitters with optical counterparts, we cross-matched the complete X-ray (having 639 unique X-ray emitters) and optical catalogs using the TOPCAT tool (Taylor 2005), applying a criterion of minimum sky distance. X-ray sources located inside the field covered by optical data and their optical counterparts are represented in Figure 3.

We chose a maximum search radius of $2^{\prime \prime}$ after carrying out several tests considering a maximum searching distance of $1^{\prime \prime}-$ $5^{\prime \prime}$ with a bin of 0.5 (Figure 4). As expected, the number of counterparts increases with the maximum search radius, but also the number of multiple matches within the error circle. Setting a search radius of $1^{\prime \prime}$, we minimize the number of multiple 


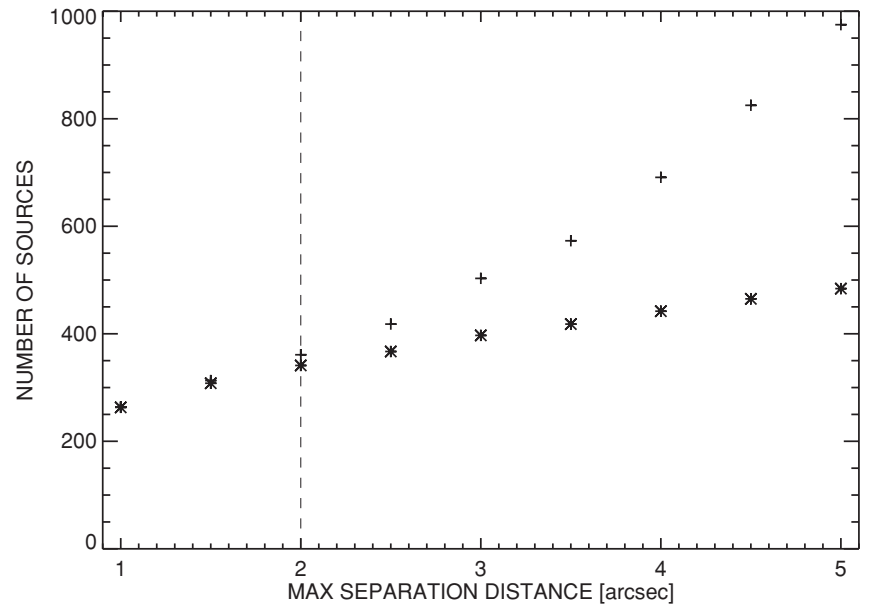

Figure 4. Number of optical counterparts as a function of the maximum search radius used for cross-matching. The number of closest sources is represented with stars. Crosses represent the total number of sources found within the search radius. The dashed line marks the adopted separation. The same value was obtained by applying the statistical methodology of de Ruiter et al. (1977).

cross-matches, but at the cost of loosing a significant number of potential counterparts. On the other hand, choosing a search radius greater than $2^{\prime \prime}$ produces a large number of multiple matches, increasing the uncertainty in counterpart identification. Therefore, we set a $2^{\prime \prime}$ search radius as an adequate compromise. This practical criterion is consistent with the more rigorous analysis performed below.

We detected 340 optical counterparts of the X-ray sources in at least one optical band. Of them, 322 are detected in the $B$ band, 332 in $V$ band, 334 in $R$ band, and 317 in $I$ band.

We carried out a quantitative analysis of the completeness ${ }^{13}$ and reliability ${ }^{14}$ of our cross-matching procedure by applying the methodology of de Ruiter et al. (1977). The separation between sources is defined by the dimensionless variable $r$ as:

$$
r=\left(\frac{\Delta \alpha^{2}}{\sigma_{\alpha}^{2}}+\frac{\Delta \delta^{2}}{\sigma_{\delta}^{2}}\right)^{1 / 2}
$$

where $\Delta \alpha$ and $\Delta \delta$ are the position differences between the $\mathrm{X}$-ray source and its optical counterpart in R.A. and decl., and $\sigma_{\alpha}^{2}$ and $\sigma_{\delta}^{2}$ are the overall astrometric errors computed as $\sigma=\left(\sigma_{\mathrm{X}}^{2}+\sigma_{\mathrm{opt}}^{2}\right)^{1 / 2}$. In this work, we assume $\sigma_{\mathrm{X}}=0.7$ and $\sigma_{\text {opt }}=0.3^{\prime \prime} . \sigma_{\mathrm{X}}$ was calculated by square adding a systematic uncertainty of 0.5 and a centroid error of 0.5 .

The likehood ratio is defined as:

$$
\operatorname{LR}(r)=\frac{d p(r \mid i d)}{d p(r \mid c)}=\frac{1}{2 \lambda} e^{\frac{r^{2}}{2}(2 \lambda-1)},
$$

where:

1. $d p(r \mid i d)$ is the a priori probability that an X-ray source and its optical counterpart have a separation between $r$ and $r+d r$ due to astrometric errors;

2. $d p(r \mid c)$ is the probability that a confusing background optical object is found in the range $r$ and $r+d r$ from the $\mathrm{X}$-ray source position; and

\footnotetext{
13 Fraction of all real associations between the X-ray and optical sources that are indeed classified as identifications.

14 Fraction of claimed identifications expected to be true counterparts
}

3. $\lambda$ measures the number of confusing objects within the error circle and depends on the optical objects density $\left(\rho_{\text {opt }}\right)$ as: $\lambda=\pi \sigma_{\alpha} \sigma_{\delta} \rho_{\mathrm{opt}}$.

An optical source can be considered as a true counterpart of an X-ray source if its LR is higher than a certain threshold value $L . L$ is obtained maximizing the sum of completeness and reliability that are, in turn, mathematically defined in terms of the probabilities defined above. In our case, $L=0.56$ and its corresponding variable is $r=2.6$, which gives us a separation distance of 2 . 0 , a value consistent with the adopted one. The completeness of our detection is $98.3 \%$ (i.e., one object lost due to the use of a particular cutoff value $L$ ), while reliability is $87.3 \%$ ( $~ 39$ potentially false matchings).

\section{MORPHOLOGICAL ANALYSIS}

\subsection{Optical Structural Parameters}

We obtained a set of structural parameters of the optical counterparts of our X-ray sample, in order to describe quantitatively their structure and morphology. In this section, we describe the procedure used to derive the parameters and to produce a morphological classification.

We used SExtractor v2.2.2 (Bertin \& Arnouts 1996) to detect and extract the sources. Details of the source extraction process are provided in Cepa et al. (2008). The version used (v2.2.2) was modified by B. Holwerda to measure the Abraham concentration index (Abraham et al. 1994). This is defined as the ratio between the integrated flux within certain radius defined by the normalized radius $\alpha$, and the total flux. We adopted $\alpha=0.3$ in our analysis in order to compare our results with those of Abraham et al. (1996). Other relevant output parameters for our study are the CLASS_STAR, which permits to roughly classify objects as compact or extended (for more information see Section 3.2), and the background that will be used later.

In addition, we used the Galaxy IMage 2D software package (GIM2D; Simard 1998; Simard et al. 2002) versions 2.2.1 and 3.1 , to perform the galaxy structural parameter decomposition. This software considers two components: a spheroid, represented by a Sérsic profile, ${ }^{15}$ and a disk represented by an exponential profile. Using the standard steps in DAOPHOT (Stetson 1987), we obtained the PSF images required for GIM2D execution. The initial parameters (including background estimation) and setup for GIM2D were determined using the images and catalogs produced by SExtractor. Figure 5 gives an example of four galaxies decomposed by GIM2D, representing for each galaxy its original image, the SExtractor segmentation image, the GIM2D model image, and the residual image (obtained after subtracting the model from the original image). We obtained from the output provided by GIM2D the following parameters ${ }^{16}$ : bulge-to-total ratio $(\mathrm{B} / \mathrm{T})$, residual parameter $(R$; Schade et al. 1995, 1996), asymmetry index (A; Abraham et al. 1994, 1996),

\footnotetext{
15 While any rigorous analysis of the structural components of an AGN host should include an additional component, namely a point-like nucleus, the small size of most of our objects compared with the seeing disk prevents from implementing such a detailed approach. Rather, we have simply assumed that the sum of the nuclear and bulge components can be roughly represented by a single Sérsic profile.

16 According to the available documentation (Simard 1998; Simard et al. 2002), GIM2D calculates the Abraham concentration at four normalized radii $\alpha=0.1,0.2,0.3$, and 0.4. (Abraham et al. 1994, 1996). Comparing the results of the concentration index obtained with the values obtained with SExtractor, for all X-ray sources from our sample, a systematic shift of $\sim 0.2$ is found toward larger values in GIM2D figures. After performing several tests we concluded that the GIM2D code performs its computations using normalized area rather than normalized radius, unlike what is stated in the documentation.
} 

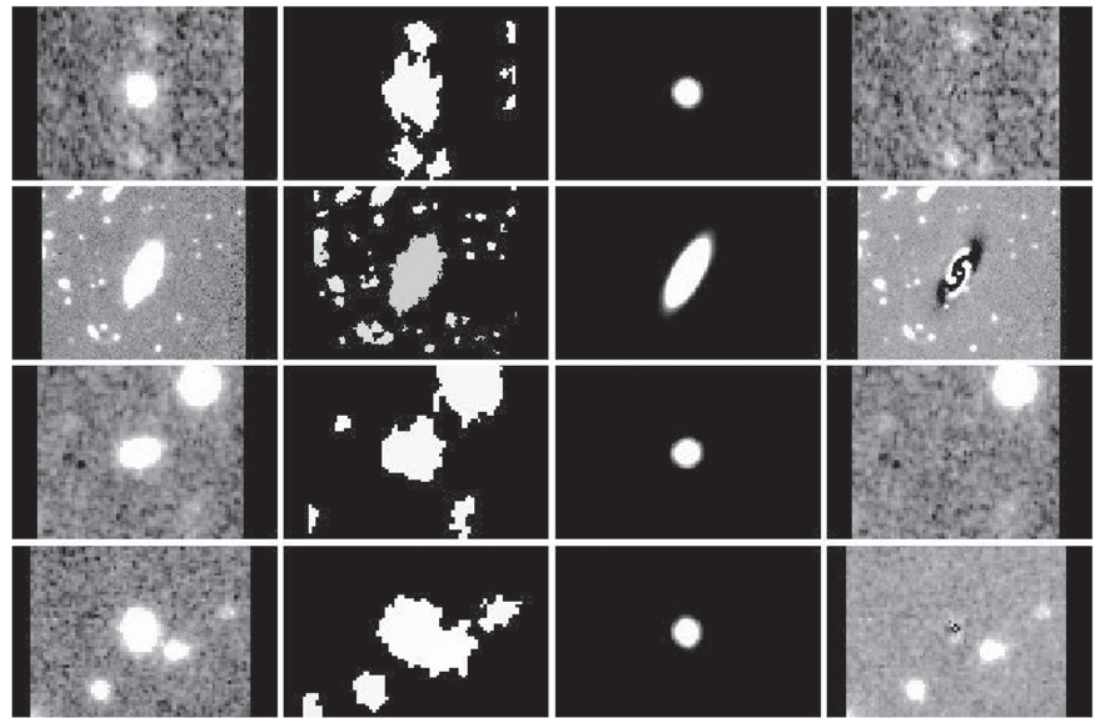

Figure 5. Four sample images of the GIM2D decomposition procedure. The first column shows subimages (obtained from the scientific image) of objects to be fitted, the second column represents the mask subimages, the third column shows the subimages obtained from the modeling, and the fourth column shows the residual images, obtained from the original image after subtracting the model.

the Sérsic index $(n)$, and the total luminosity $\left(L_{\mathrm{tot}}\right)$. The residual parameter and the asymmetry index quantify the galaxy irregularity, by measuring the deviation from the simple, symmetric model (e.g., spheroid + disk), using as input the residual and original images, respectively. For all output parameters (with the exception of the asymmetry index), GIM2D also calculates lower and upper limits at $99 \%$ confidence level. In order to estimate the accuracy of the best fit parameters, we have looked at the deviation, computed as the maximum between the best value and the upper or lower limits. Figure 6 presents the median value of this deviation as a function of $\mathrm{X}$-ray source isophotal area for three derived parameters: total luminosity, B/T flux ratio, and Sérsic index. Regarding this, the $\mathrm{B} / \mathrm{T}$ parameter and Sérsic index have revealed inaccurate when applied to faint and small objects $(\lesssim 300$ pixel, while the seeing disk (FWHM) area is about 30 pixels). Therefore, we have decided to avoid using these parameters for the morphological classification.

In practice, we observed that the code either fails or produces unreliable output parameters in a number of cases: (1) when the source $R$ magnitude is less than 18 or larger than 24; (2) when the isophotal area is very small (usually less then 90 pixels); (3) when the central object is surrounded by close neighboring sources or nearby bright companions; and (4) when the source is close to the frame boundaries.

\subsection{Morphological Classification}

The selection of compact objects (QSOs, faint host AGNs, or active stars) has been accomplished by using the SExtractor CLASS_STAR output parameter. The computed value depends on the seeing, the peak intensity of the source and its isophotal area. The parameter ranges between 0 (extended object like a galaxy) and 1 (point-like object). A source has been deemed as compact if its CLASS_STAR parameter is larger than 0.9.

To determine the morphology of extended objects (i.e., not selected as compact according to the criterion above), we analyzed four parameters: Abraham concentration index (C), Abraham asymmetry index (A), B/T ratio, and residual parameter $(R)$. We carried out the analysis in three different bands $(B, V$, and $R)$ in order to determine the most reliable parameters, and the best band for morphological classification.

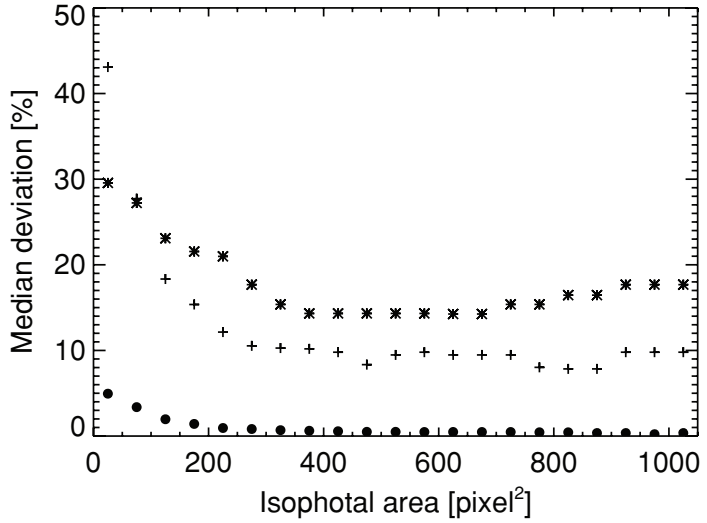

Figure 6. Evolution of the median error of the bulge-to-total ratio (B/T; crosses), Sérsic index $n$ (stars), and total flux (filled circles) $L_{\text {tot }}$ as functions of the isophotal area. Deviations are evaluated as explained in Section 3.1.

We excluded the $I$ band in the investigation of the optimum parameter space in order to avoid potential problems caused by fringing effects (monochromatic interference in the CCD generated by night sky line emission sources; Cepa et al. 2008).

We first classified each object using the set of parameters mentioned above, separately for each band, considering the results from previous works as limits between different morphological types. We used the results of Simard (1998) and Im et al. (2002) to obtain preliminary morphology estimates using the $\mathrm{B} / \mathrm{T}$ parameter, Schade et al. (1995) results for the residual parameter, and Abraham et al. (1996) for the classification using the concentration and asymmetry indexes. In practice, we found that, in many cases, different parameter sets lead to a different classifications of a given object even using the same band. In order to have an independent evaluation criterion, we also carried out a visual classification of the objects. Despite its subjectiveness, this procedure can yield highest quality results when applied to bright and extended objects, thus being a convenient method to test the reliability of the structural parameters that will be, in turn, applied to the analysis of fainter objects. The visual classification has been accomplished by inspection of the images and isophotal contour diagrams. Figure 7 shows the examples of six 

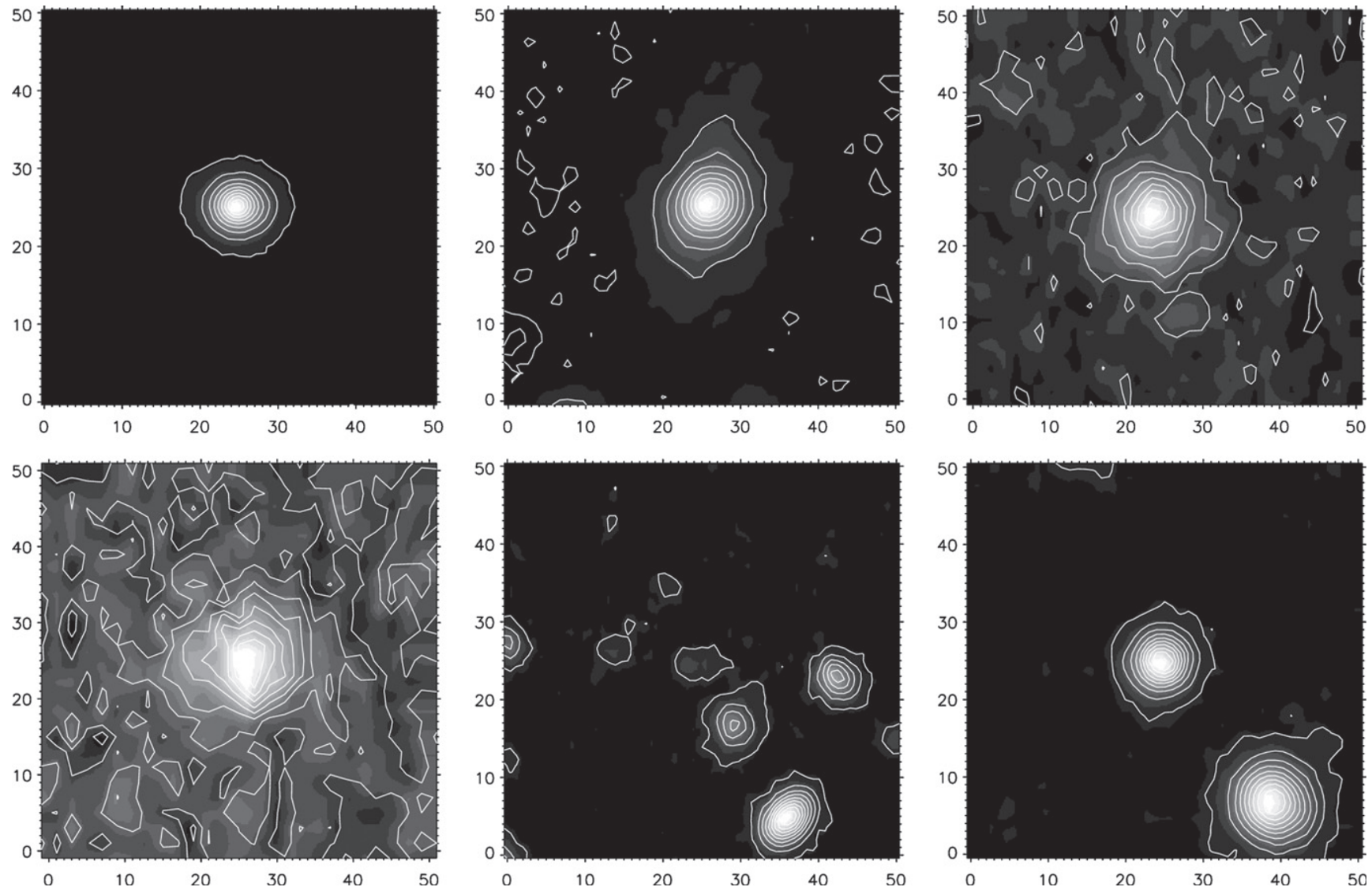

Figure 7. Examples of different Hubble-type galaxies and their contour diagrams; Top row—Left panel: group I (E/S0), Center panel: group II (early-type spiral), Right panel: group III (spiral); Bottom row: Left panel, group III (spiral), Center panel: group IV (Irr), Right panel: compact object (C $\geqslant 0.7$ or CLASS_STAR > 0.9). All objects are located in the center of the images.

isophotal contour diagrams for different Hubble-type galaxies. For the dimmest and smallest objects of our sample (galaxies with the magnitude $R>24$ and isophotal area $<100$ pixels), the profiles and isophotal contours are too noisy to get an acceptable result by means of a simple visual inspection.

From this comparison, we conclude that for our sample, characterized by faint objects (typically $R \gtrsim 22$ ) with small isophotal area $(\lesssim 300$ pixel, while the seeing FWHM disk area is about 30 pixels), the most reliable parameter is the concentration index $\mathrm{C}$, combined either with the asymmetry index or the residual parameter. Then, in this work, we based our morphological classification in these parameters. The $R$ band has been used because the largest part of optical counterparts have been found in this band, and the achieved signal-to-noise ratio $(\mathrm{S} / \mathrm{N})$ is generally larger. $B, V$, and $I$ bands have been used to perform the morphological classification of $\sim 5 \%$ of objects not detected in the $R$ band, or detected in this band but with very low $\mathrm{S} / \mathrm{N}$.

Based on the classification from visual inspection of the brightest sample objects, and upon comparison with previous works (e.g., Abraham et al. 1996, see references above), we created five categories or groups associating ranges of values of $\mathrm{C}$ with Hubble types:

$0: C \geqslant 0.7$ or CLASS_STAR $\geqslant 0.9 \rightarrow$ Compact object;

I: $0.45 \leqslant \mathrm{C}<0.7 \rightarrow \mathrm{E}, \mathrm{E} / \mathrm{S} 0$, and S0;

II: $0.3 \leqslant \mathrm{C}<0.45 \rightarrow$ SO/SOa-Sa;

III: $0.15 \leqslant \mathrm{C}<0.3 \rightarrow$ Sab-Scd;

IV: $\mathrm{C}<0.15 \rightarrow$ Sdm-Irr.

Group I is populated by elliptical and lenticular galaxies, whose profiles do not show any trace of disk or spiral arms (see Figure 7), group II includes very early spiral type galaxies, which show signs of spiral arms. Group III comprises spiral galaxies while group IV includes late-type spirals and irregular galaxies, which show almost no regular form in their profiles. All galaxies, even those in the first group (elliptical and lenticular) have a concentration index $\mathrm{C} \leqslant 0.7$, while all objects with $\mathrm{C}>0.7$ have been classified by SExtractor as compact ones, with a CLASS_STAR parameter $>0.9$.

In Figure 8, we depict the Abraham asymmetry versus concentration index diagram segregated according to the morphology determined using the visual classification for the brightest objects, and the structural parameters (in most cases $\mathrm{C}$ combined with A) for the fainter ones. Comparing Figure 8 with that shown in Abraham et al. (1996), it can be seen that in our case there is a small shift of the concentration index toward smaller values. This can be explained considering the influence of the atmospheric seeing conditions. SExtractor does not apply any correction on the computed parameters for this effect, and as the seeing induced PSF increases, both concentration and asymmetry indexes tend to decrease, as confirmed by our simulations carried out by convolving simple galaxy models with seeing PSFs of different widths. Since the Abraham et al. (1996) diagram has been obtained from HST data, such differences are expected.

The morphological analysis described above has been applied to the complete sample of $340 \mathrm{X}$-ray objects with optical counterpart (see Figure 9). Of those, 333 were classified using the $R$ band, 4 using the $V$ band, and finally 3 by means of $B$-band data. The largest group (143 sources) is composed by compact objects (QSOs, faint host AGNs, and stars) followed by group 


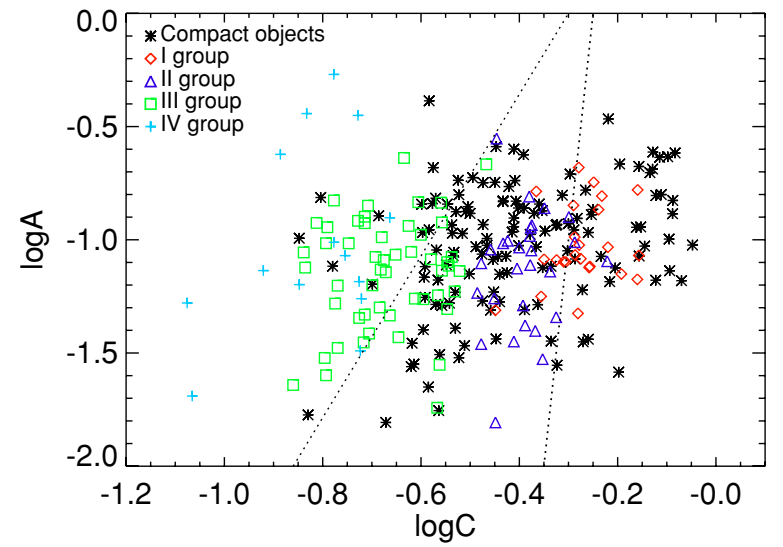

Figure 8. Morphological classification diagram combining the Abraham asymmetry index (A) vs. Abraham concentration index (C). Diamonds represent E and S0 galaxies, triangles S0/S0a-Sa galaxies, squares young spiral galaxies (Sab-Sbcd), and crosses late-type galaxies (Sdm-Irr). Compact objects are represented by star symbols. Dotted lines separate the regions of elliptical, spiral, and peculiar galaxies (from right to left) obtained by Abraham et al. (1996). There is a small shift of our concentration and asymmetry indexes toward smaller values due to the influence of the atmospheric seeing conditions.

(A color version of this figure is available in the online journal.)

III (73 galaxies). 57 objects are found either in group I or II, having outstanding bulge component, and signs of a formed disk and spiral arms. Only 33 galaxies are found to belong to the IV group. Finally, we detected seven close pairs, whose possible physical relation would be confirmed once photometric redshifts are computed (J. Cepa et al. 2010, in preparation, Paper III). Due to the high noise level, or the presence of other objects close to the observed ones, the classification uncertainty for some of the objects is quite high, and it was not possible to determine the morphological group: three galaxies could be either in group II or III and other three either in group III or IV. For 21 sources, it was not possible to determine the morphology since they are at the detection limit.

\section{RELATIONSHIP BETWEEN X-RAY AND OPTICAL STRUCTURAL PROPERTIES}

\subsection{X-ray Classification}

A widely used way to classify the X-ray emitters is based on the X-ray-to-optical flux ratio $(\mathrm{X} / \mathrm{O})$. The typical value of $\mathrm{X} / \mathrm{O}$ ratios for X-ray selected AGN (both unobscured or X-ray type 1 and obscured or X-ray type 2) ranges between 0.1 and 10 (e.g., Fiore et al. 2003). For X-ray selected type 1 AGN, the typical value is $\mathrm{X} / \mathrm{O} \simeq 1$ (Alexander et al. 2001; Fiore et al. 2003). At high $X / O$ flux ratios (well above 10), we can find $\mathrm{X}$-ray type 1 and X-ray type 2 AGNs, as well as high- $z$ highluminosity obscured AGN (type 2 QSOs), high- $z$ clusters of galaxies and extreme BL Lac objects. Finally, the $\mathrm{X} / \mathrm{O}<0.1$ region is typically populated by coronal emitting stars, normal galaxies (both early-type and star-forming), and nearby heavily absorbed (Compton thick) AGNs (e.g., Comastri et al. 2002; Civano et al. 2007).

Moreover, different works have demonstrated how a Xray color-based analysis can be used to constrain models of absorption in the AGN population, without requiring complete optical spectroscopic follow-up (e.g., Mainieri et al. 2002; Della Ceca et al. 2004; Perola et al. 2004; Caccianiga et al. 2004; Dwelly et al. 2005; Hasinger 2008). In optically identified samples, the AGNs with and without broad emission lines

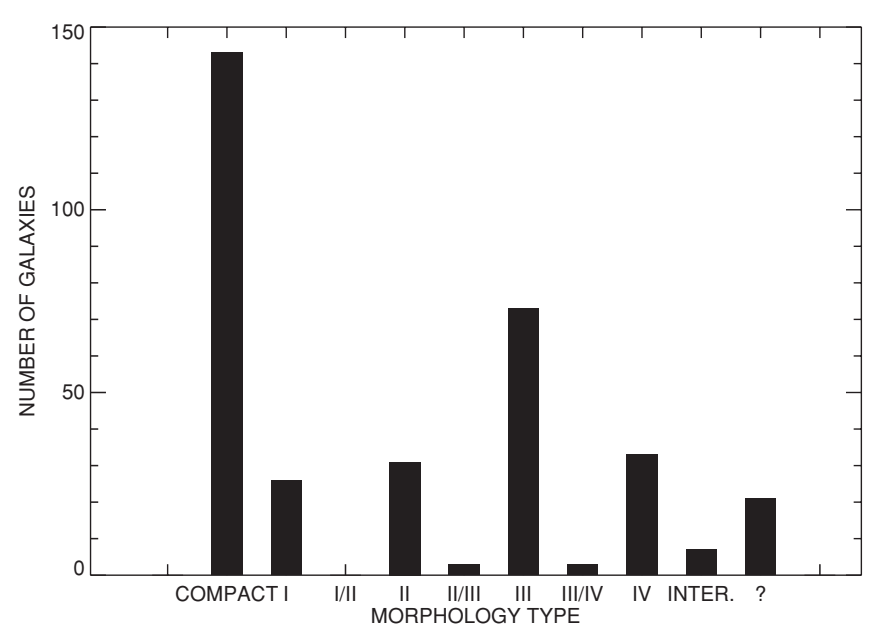

Figure 9. Histogram represents the final morphological classification of the GWS X-ray emitters with optical counterparts. Different values of the Abraham concentration index correspond to different groups I-IV (see the text). A group of possible interacting objects has been detected; a group of sources (marked with “?”) remained unidentified due to their very low $\mathrm{S} / \mathrm{N}$.

are seen to have different $\mathrm{X}$-ray hardness ratio values. It is shown that the cut of $\mathrm{HR}(2-4.5 \mathrm{keV} / 0.5-2 \mathrm{keV})>-0.35$ can efficiently discriminate between optical type 1, and type 2 AGN sample (Caccianiga et al. 2004; Della Ceca et al. 2004). These results are also in agreement with those obtained by Dwelly et al. (2005), who shown, by using extensive Monte Carlo simulations, that approximately the same cut will select efficiently the majority of the most heavily absorbed sources $\left(\log N_{H}>22.5\right)$.

We have applied a simple criterion for performing a coarse $\mathrm{X}$-ray type classification of our sample objects, based on diagnostic diagrams relating $\mathrm{X} / \mathrm{Os}$ to HRs. Using the $X M M$ Newton Bright Serendipitous Survey data, Della Ceca et al. (2004) found that, when plotting the already mentioned HR(2$4.5 \mathrm{keV} / 0.5-2 \mathrm{keV}$ ) (equivalent to our $\mathrm{HR}_{1}^{\prime}$ ) against $\mathrm{X} / \mathrm{O}$ flux ratio, computed as the ratio of the observed $\mathrm{X}$-ray flux in the $0.5-4.5 \mathrm{keV}$ (soft+hard2 in our case) energy range, and the optical $R$-band flux, most $(85 \%)$ of X-ray type 1 AGNs, optically identified with broad emission lines, are tightly packed in a small rectangular region of the diagram, while X-ray type 2 AGNs, optically identified as narrow line AGNs, tend to populate a wide area of the diagram (toward harder values of the hardness ratio). We applied the same approach to our sample, combining the $\mathrm{HR}_{1}^{\prime}$ hardness ratio with the $\mathrm{X} / \mathrm{O}$ ratio, where the optical flux $F_{R}$ has been derived from the Petrosian magnitudes in the $R$ band. The resulting diagram, which also includes host morphology information, is shown in Figure 10. According to the previous discussion, all objects having $\mathrm{X} / \mathrm{O}>0.1$ and $\mathrm{HR}_{1}^{\prime}<-0.35$ have been classified as $\mathrm{X}$-ray type 1 (unobscured), while objects with $\mathrm{X} / \mathrm{O}>0.1$ and $\mathrm{HR}_{1}^{\prime}<-0.35$ have been classified as X-ray type 2 (obscured) AGNs. We find that a large fraction of our objects, $63 \%,{ }^{17}$ fall inside the region of X-ray type 1 AGNs. The dashed-line box indicates the locus of the X-ray type 1 region according to Della Ceca et al. (2004). We consider this box as the "highest probability" region for finding a X-ray type 1 AGN. 51\% of the sample is placed within this box. Regarding morphology, we do

\footnotetext{
17 All fractions given here are relative to the total number of X-ray objects with optical counterpart, and detected in both soft, and hard 2 energy bands such as $\mathrm{HR}(2-4.5 \mathrm{keV} / 0.5-2 \mathrm{keV})$ can be derived.
} 


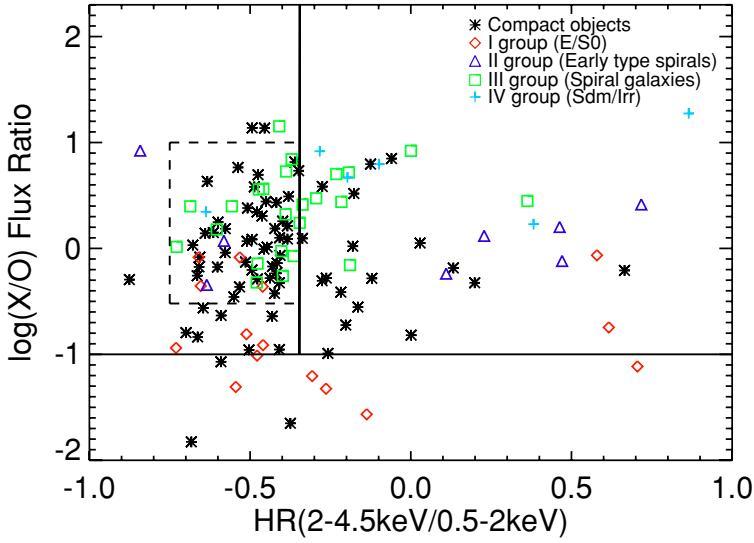

Figure 10. Relationship between the $X / O$ ratio and $\mathrm{HR}(2-4.5 \mathrm{keV} / 0.5$ $2 \mathrm{keV}$ ), for the different morphological types. Solid lines separate X-ray type 1 (unobscured) and $\mathrm{X}$-ray type 2 (obscured) regions and the area with $\mathrm{X} / \mathrm{O}<0.1$, where stars, normal galaxies, and Compton thick AGNs can be found (Fiore et al. 2003). The dashed-line box represents the limits obtained by Della Ceca et al. (2004) where $~ 85 \%$ of their spectroscopically identified AGNs with broad emission lines (X-ray type 1 AGNs) have been found. In our case, a fraction of $\sim 51 \%$ of the sample can be found inside this box

(A color version of this figure is available in the online journal.)

not find evidence of any relationship between X-ray and morphological types, since we did not obtain any clear separation of X-ray properties based on morphological type. Nevertheless, we can see that the majority of objects identified as compact $(65 \%)$ are placed in the X-ray type 1 region. It is also interesting to highlight the relationship between the $\mathrm{X} / \mathrm{O}$ ratio and the host galaxy morphology, with early-type galaxies having generally lower values for $\mathrm{X} / \mathrm{O}$ ratio and an opposite behavior of late-type objects. Finally, we find that only about $7 \%$ of our sample objects are placed in the lower region of the diagram (i.e., that typical of stars/normal galaxies/Compton-thick AGN).

We also represented the $X / O$ ratio with respect to $H R_{1}$, yielding a basically identical distribution as that described above, and with respect to $\mathrm{HR}_{2}$ and $\mathrm{HR}_{2}^{\prime}$, observing that in these two cases it is more difficult to get a clear separation between different X-ray/morphological types; this behavior is expected since hardness ratios computed from the hard and vhard bands are less sensitive to absorption than those involving the soft band, which is one of the main criteria for the separation between X-ray type 1 and type 2 AGNs.

\subsection{X-ray and Optical Properties}

\subsubsection{The Clustering of $X$-ray Emitters and Extended Optical Counterparts}

The study of large-scale environmental conditions of AGN host galaxies is useful not only for understanding the differential properties of the AGN phenomena (e.g., the growth of supermassive black holes), but to trace the formation and evolution of such galaxies and the structures where they reside. Despite the relevance of X-ray selected AGNs, the clustering properties of their optical counterparts still remain quite unknown (Basilakos et al. 2005, and references therein). Such properties are essential in the solution of these problems because AGN hosts can be more easily detected in large cosmological volumes compared with normal galaxies and, in the other hand, the clustering features provide clues about the links between AGN activity and host properties (e.g., typical mass of dark matter halos where
AGN reside, as well as likely lifetimes of the latter). In this sense, and taking into account the characteristics of the X-ray source catalog presented in this work, we have calculated the two-point angular correlation function ( $2 p-A C F)$ for significant subsamples of optical counterparts.

The 2p-ACF, $\omega(\theta)$, gives the excess of probability, with respect to a random homogeneous distribution, of finding two sources in the solid angles $d \Omega_{1}$ and $d \Omega_{2}$ separated by an angle $\theta$, and it is defined as

$$
d P=\mathcal{N}^{2}[1+\omega(\theta)] d \Omega_{1} d \Omega_{2},
$$

where $\mathcal{N}$ is the mean number density of cataloged sources. To measure the $2 \mathrm{p}-\mathrm{ACF}$, we have followed the same procedure as that described in Cepa et al. (2008), adopting the estimator of Landy \& Szalay (1993), which can be written in the form

$$
\omega\left(\theta_{i}\right)=\frac{D D-2 D R+R R}{R R},
$$

where $D D=N_{s s}\left(\theta_{i}\right)$ is the fraction of possible source-source pairs counted in $i$-bins over the angular range studied and $D R=$ $\left[\frac{\left(N_{g}-1\right)}{2 N_{r}}\right] N_{g r}\left(\theta_{i}\right), R R=\left[\frac{N_{s}\left(N_{s}-1\right)}{N_{r}\left(N_{r}-1\right)}\right] N_{r r}\left(\theta_{i}\right)$ are the normalized counts of source-random and random-random pairs in these bins, respectively. To make the random catalogs, we placed $\left(200 \times N_{\text {source }}\right)$ random points by following the X-ray source angular distribution. However, the source density is affected by several instrumental biases such as detector gaps and sensitivity variations due to survey regions with different exposure times. To account for these effects, we made normalized sensitivity maps from the effective exposure times and source distributions for each band used in this section (soft and hard2). The raw maps were then modeled using a minimum-curvature algorithm (Briggs 1974). Next, the uniform density random catalogs were convolved with the corresponding sensitivity map to obtain differential number counts in the surveyed area. In the case of the catalog having sources with $\mathrm{HR}_{1}^{\prime}$, we adopt the sensitivity map used for the hard2 source one. The smoothed footprint of each sensitivity map can be seen in Figure 11.

We measure $\omega\left(\theta_{i}\right)$ in $i$-logarithmic bins of width $\Delta(\log \theta)=$ 0.2 in the angular scales $0.24 \leqslant \theta \leqslant 24^{\prime} .0$. The lower limit in angular separation has been set to match the minimum detection scale defined in the Section 2.1.2. The formal error associated with the $2 \mathrm{p}$-ACF measured is the Poissonian estimate $\sigma_{\omega}^{2}=\frac{1+\omega\left(\theta_{i}\right)}{D D}$. The $2 \mathrm{p}$-ACF binned measures were fitted using the $A_{\omega}\left(\theta^{1-\gamma}-C\right)$ power law. We adopted a fixed canonical slope $\gamma=1.8$ (the so called "comoving clustering model") and $\mathrm{C}$ is defined as

$$
C=\frac{\sum N_{r r}(\theta) \theta^{-0.8}}{\sum N_{r r}(\theta)},
$$

corresponding to the numerical estimation of the "integral constraint" (Peebles 1980). As in Miyaji et al. (2007), we use the normalization $A$ as the fitting parameter rather than the correlation length $\theta_{c}=A^{1 /(\gamma-1)}$, since the former gives better convergence of the fit.

From the whole catalog, we have selected the soft $(0.5-2 \mathrm{keV})$ and hard2 (2-4.5 keV) sources (given their similar depths in the survey), the X-ray objects with $\mathrm{HR}_{1}^{\prime} \neq 0$ and the fraction of $\mathrm{X}$ ray sources with optical counterparts. Table 2 summarizes the basic results of the correlation analysis for these subsamples. In all cases, we have obtained significant positive clustering signal. Regarding the soft band sample, the correlation length $\theta_{c}$ derived from $A_{\omega}$ is consistent with the obtained by Basilakos 
Table 2

Clustering Properties of the X-ray Selected Sources

\begin{tabular}{lccccc}
\hline \hline \multicolumn{1}{c}{ Subsample } & $N_{\text {source }}$ & $\begin{array}{c}\theta_{\min }-\theta_{\max } \\
(\operatorname{arcmin})\end{array}$ & $\begin{array}{c}A_{\omega}(1-\gamma=-0.8) \\
\times 10^{-3}(\mathrm{deg})\end{array}$ & $\chi^{2}$ & $\omega\left(1^{\prime}\right)$ \\
\hline soft $(0.5-2 \mathrm{keV})$ & 465 & $0.24-24.0$ & $6.99 \pm 0.68$ & 0.030 & $0.15 \pm 0.02$ \\
hard2 $(2-4.5 \mathrm{keV})$ & 266 & $0.24-24.0$ & $9.19 \pm 1.28$ & 0.107 & $0.20 \pm 0.03$ \\
HR $(2-4.5 \mathrm{keV} / 0.5-2 \mathrm{keV})$ & 190 & $0.24-24.0$ & $9.68 \pm 1.30$ & 0.109 & $0.21 \pm 0.03$ \\
Optical counterparts $(I \leqslant 23)$ & 104 & $0.60-24.0$ & $19.99 \pm 3.32$ & 0.096 & $0.42 \pm 0.07$ \\
\hline
\end{tabular}

Notes. $N_{\text {source }}$ is the number of data points used in the 2p-ACF estimation and $A_{\omega}$ is the best-fit value in the separation range given by $\theta_{\min }-\theta_{\max }$, assuming a power law $\omega(\theta)=A_{\omega} \theta^{(1-\gamma)=-0.8}$. The goodness-of-fit statistics $\left(\chi^{2}\right)$ and the fitted $2 \mathrm{p}-\mathrm{ACF}$ at $1^{\prime}\left[\omega\left(1^{\prime}\right)\right]$ are given in the final columns. The errors represent $1 \sigma$ uncertainties.
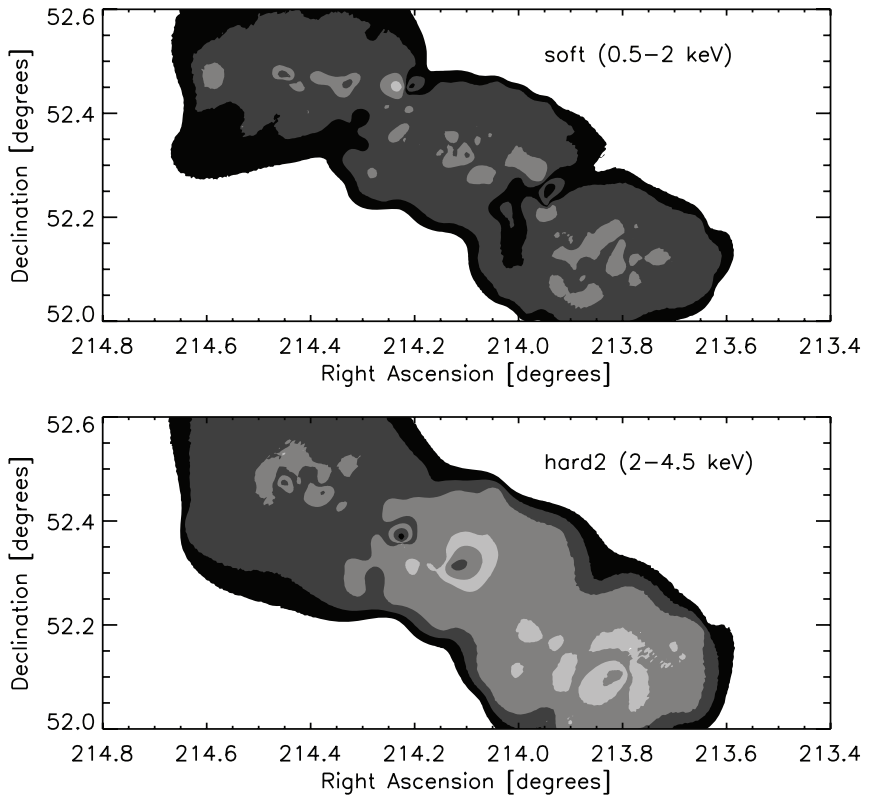

Figure 11. Smoothed sensitivity maps in the soft $(0.5-2 \mathrm{keV})$ and hard $2(2-$ $4.5 \mathrm{keV}$ ) bands, used to convolve the uniform density random catalogs. The sensitivity dimension is normalized to the maximum exposure time in each band. Contours are linearly binned and lighter regions correspond to higher sensitivities.

et al. (2005; XMM-Newton LSS), and is $\sim 2 \sigma$ larger than the values obtained by Gandhi et al. (2006); Carrera et al. (2007); and Ueda et al. (2008). In the specific case of the hard2 band, we only found in the literature the $2 \mathrm{p}-\mathrm{ACF}$ estimations of Miyaji et al. (2007), valid for X-ray sources detected in the XMMNewton observations of the COSMOS field, and corresponding to their MED band. Our fitted correlation length is $\sim 4.3$ times larger, although our sample is a factor 2 deeper.

In Figure 12, our 2p-ACF measurements with the best-fit $A_{\omega} \theta^{-0.8}$ power law in soft and hard2 bands (panels a and $\mathrm{b}$, respectively) are represented. It is noticeable that the clustering amplitude obtained in both bands is similar. Thus, in the case of the Groth field, we would not expect redshift or spatial clustering differential effects in the soft and hard 2 band samples. Interestingly, an absence of correlation in differently defined hard bands has been reported by Gandhi et al. (2006) and Puccetti et al. (2006); nevertheless, Yang et al. (2003) and Basilakos et al. (2004) not only yield a positive correlation amplitude, but the hard-band signal is several times that of the soft band. Other results with similar correlation lengths in both bands are given by Yang et al. (2006); Carrera et al. (2007); and Miyaji et al. (2007).
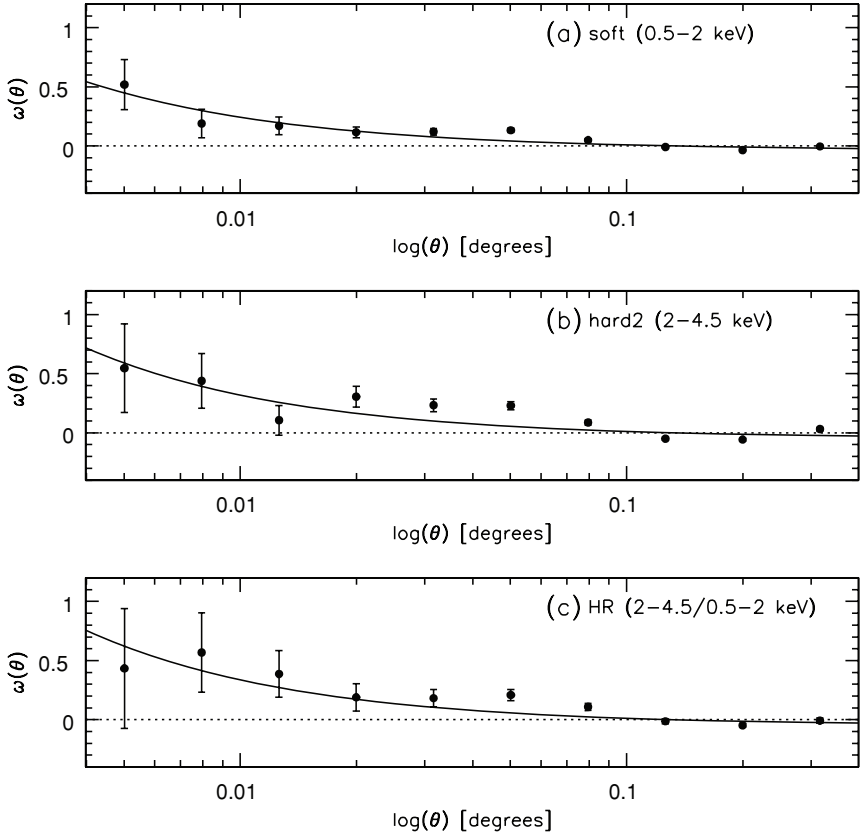

Figure 12. 2p-ACF estimates of the X-ray sources detected in the soft band (panel a), hard2 band (panel b), and HR as defined in Equation (1) (panel c). The continuous line represents the $A_{\omega} \theta^{(1-\gamma)=-0.8}$ power law fitted to the data.

In the panel $\mathrm{c}$ of Figure 12, the 2p-ACF estimations for a $\mathrm{HR}(2-4.5 \mathrm{keV} / 0.5-2 \mathrm{keV})$ selected sample are represented. The observed clustering amplitude is slightly larger than that of soft or hard2 band. Moreover, the whole $\mathrm{HR}_{1}^{\prime}$ subsample was decomposed using the boundary referred in Section 4.1 to separate X-ray type 1 from X-ray type 2 populations and a positive, very similar clustering signal from both subsamples, emerged from the 2p-ACF estimations, despite that their goodness-of-fit statistics are worse than the tabulated value for $\mathrm{HR}_{1}^{\prime}$ subsample in a factor $\sim 1.8$ because of the small size of the subsets.

Finally, we measured the 2p-ACF of the optical counterparts of X-ray sources in order to compare the clustering behavior with those that come from the analysis of galaxy populations in the same field. For a reliable comparison is necessary to reject the compact objects (i.e., with CLASS_STAR > 0.9) from the optical counterparts catalog, as well as all objects fainter than a limiting magnitude. We have selected the peak of the apparent brightness distribution in the $I$ band as such limiting magnitude. Thus, $X$-ray counterparts with $I>23$ were ruled out, remaining 104 extended objects, which can be regarded as AGN host candidates of the corresponding X-ray sources.

In spite of this skinny sample, it is possible to obtain some interesting lines of thought about the X-ray selected AGN 
clustering. It is suggested that the typical environments of AGNs are not different from those of quiet galaxies in general, at redshift ranges above $\mathrm{z} \sim 0.4$ (e.g., Grogin et al. 2003; Waskett et al. 2005). Nevertheless, Coil et al. (2004) and Gilli et al. (2005) have argued that the AGN are preferentially hosted by early-type galaxies at a typical redshift of $\sim 1$. In this way, Basilakos et al. (2004) found that clustering length values of hard X-ray objects are comparable with those of extremely red objects (EROs) and luminous radio sources. More recently, Georgakakis et al. (2007) demonstrated that X-ray-selected AGNs at zsim 1, in the AEGIS field, avoid underdense regions at $99.89 \%$ of significance.

In Figure 13, we have depicted the $2 \mathrm{p}-\mathrm{ACF}$ of the optical counterparts selected as described above. The $2 \mathrm{p}$-ACFs of the Groth field galaxy sample with $I \leqslant 23$ only and with $V-I>3$ $(I \leqslant 24)$ from Cepa et al. (2008) are over plotted. At least in the range $0.01 \leqslant \theta \leqslant 0.1$, the clustering signal produced by the optical counterparts of our X-ray sources is $1 \sigma-2 \sigma$ stronger than the corresponding to the $I \leqslant 23$ galaxy population. In terms of fitted correlation length, the $\theta_{c}$ (X-ray) $(1-\gamma=-0.8)$ is $~ 30$ times larger than that of the Groth field galaxies, but only $\sim 2.3$ times larger than the maximum correlation length of the $V-I>3$ galaxies in the same field. On the other hand, using the optical data from the counterparts catalog, we found that the median $V-I$ observed color is 1.86 and most of $65 \%$ of the optical counterparts have an observed color $V-I>2$. This means that a significant population of our optical counterparts is a composition of red and very red galaxies, whereas its clustering signal tends to reproduce that associated with such galaxies, which drive the $\sim 10 \times$ clustering excess found by Cepa et al. (2008). More than a 55\% of the optical counterparts belong to the morphological categories I and II, as defined in the Section 3.2.

\subsubsection{X/O Ratio versus Optical Colors}

We combined the $\mathrm{X} / \mathrm{O}$ ratio with optical colors $(B-R$ and $B-I$ ) for the different morphological groups and X-ray types, as shown in Figure 14. We observe that compact and late-type objects tend to show bluer colors, while early-type galaxies tend to present redder colors and also lower $\mathrm{X} / \mathrm{O}$ values, as expected. On the other hand, there is a mild tendency for X-ray type 1 AGNs to show bluer colors than those found in X-ray

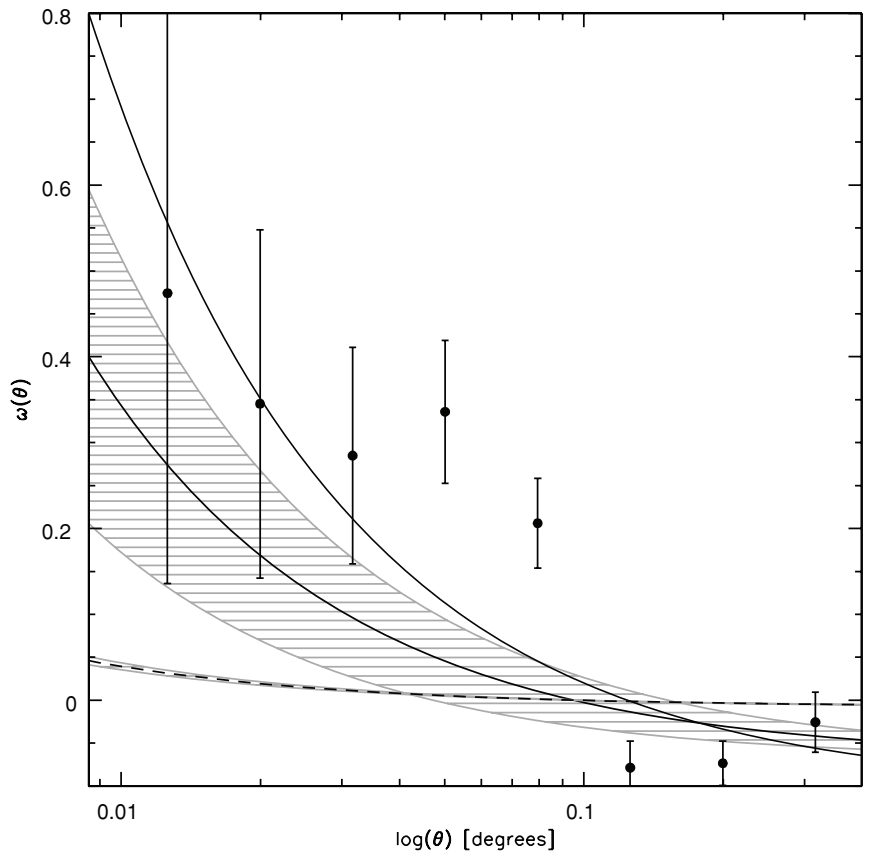

Figure 13. 2p-ACF estimation for the optical extended (CLASS_STAR $\leqslant 0.9)$ counterparts with $I$ magnitude brighter than 23 (solid circles). The continuous thick line represents the $A_{\omega} \theta^{(1-\gamma)=-0.8}$ power law fitted to the data. The dashed line represents the 2 p-ACF estimation for galaxies with $I \leqslant 23$ in the Groth field, whereas the continuous thin line does it for the $V-I>3$ color selected galaxies with $I \leqslant 24$, both extracted from Cepa et al. (2008). The shaded regions represent fitting errors of $1 \sigma$.

type 2, as already observed in local universe samples (e.g., Yee 1983; Sánchez-Portal et al. 2004). The $B-R$ average value for $\mathrm{X}$-ray type $1 \mathrm{AGNs}$ is 1.0 and 1.4 for type 2 , with $\sigma=0.5$ and 0.6 , respectively. Using the Kolmogorov-Smirnov test, the two distributions are statistically different with a significance level of $99 \%$.

Our morphological classification is consistent with the recent results (e.g., Sánchez et al. 2004; Silverman 2008; Bundy et al. 2008; Gabor et al. 2009) suggesting that galaxies hosting AGNs predominantly represent a morphological transitional population, shifting from blue to red colors on the colormagnitude diagrams.
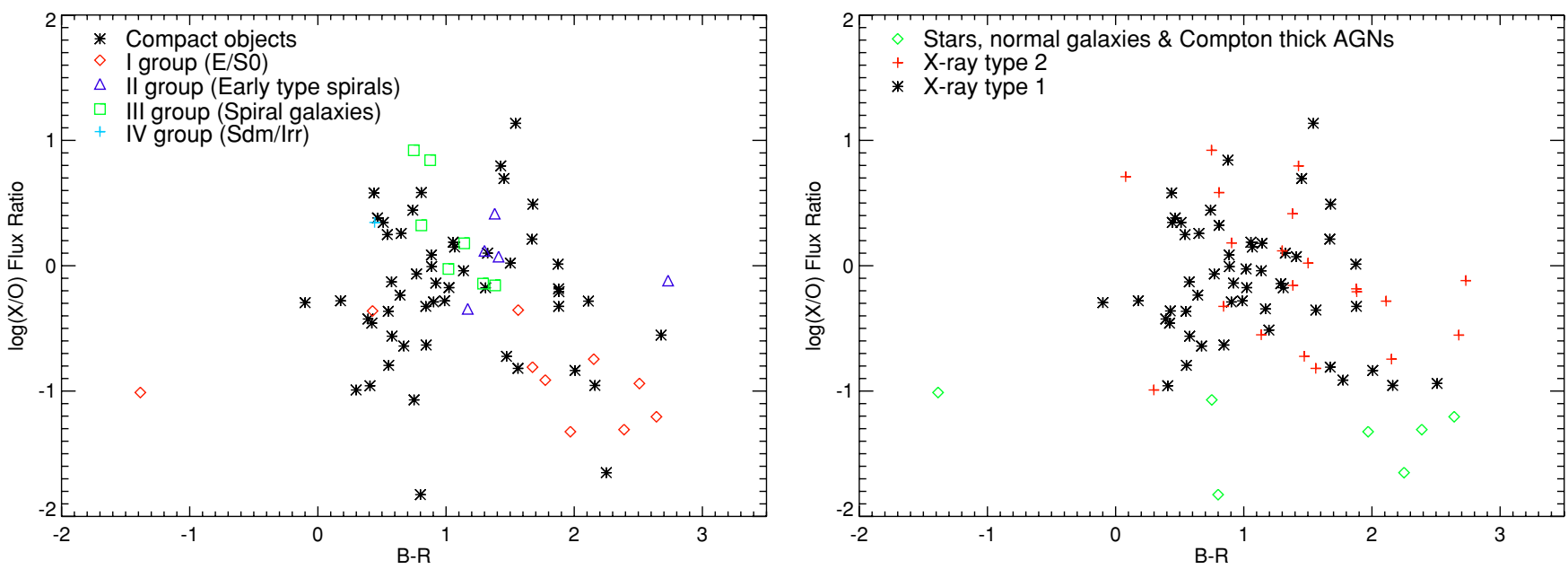

Figure 14. $\log (\mathrm{X} / \mathrm{O})$ flux ratio vs. $B-R$ color for different morphological (left panel) and X-ray types (right panel).

(A color version of this figure is available in the online journal.) 

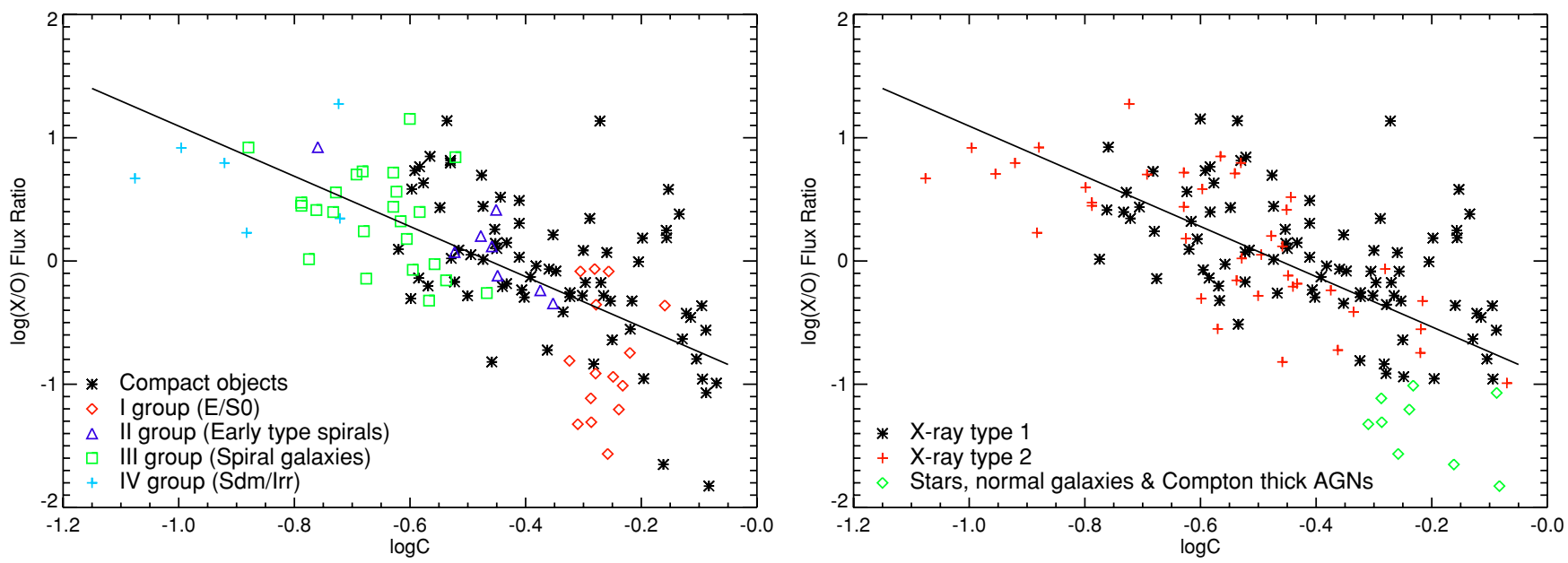

Figure 15. Relationship between $\mathrm{X} / \mathrm{O}$ and the Abraham concentration index $\mathrm{C}$ for different morphological (left panel) and X-ray types (right panel). A clear anticorrelation is observed.

(A color version of this figure is available in the online journal.)

\subsubsection{X/O Ratio versus Galaxy Light Concentration}

When representing the $\mathrm{X} / \mathrm{O}$ flux ratio versus the concentration index $\mathrm{C}$, we have found an anticorrelation between these two parameters (Figure 15), with a correlation coefficient of $\sim-0.5$ and a correlation significance level $>99 \%$ according to Spearman and Kendall statistics. The first order polynomial function has been fitted with a slope of $-2.03, \log \mathrm{X} / \mathrm{O}$-intercept of -0.94 , and a standard deviation of $\sim 0.5$. This result is consistent with that referred above and depicted in Figure 10, that is the tendency of early-type galaxies to have lower than average $\mathrm{X} / \mathrm{O}$ values and the opposite behavior in late-type ones. As seen in Figure 15, all morphological groups, including compact objects, tend to follow the same relation. Comparing different $\mathrm{X}$-ray types, we have found that both X-ray type 1 and X-ray type 2 AGNs follow the same distribution (see Figure 15).

This anticorrelation was already observed in the population of AGNs of the Subaru/XMM Deep Survey (SXDS) field, ${ }^{18}$ and is described in Pović et al. (2009, hereafter P09). This anticorrelation was found to be significant in a wide range of photometric redshifts, with a similar first grade polynomial function fitting in different redshift bins. In P09, we studied different bias effects, namely redshift and bandpass shift, obscuration, nuclear pointlike source influence, apparent brightness and size, and seeing, which could affect measurements on both parameters, but especially on concentration index. Moreover, we performed further tests of the existence of an anticorrelation between $\mathrm{X} / \mathrm{O}$ and $\mathrm{C}$ using a galaxy mock catalog built from the Millennium-II Simulations (Boylan-Kolchin et al. 2009, and references therein) database finding a very good correspondence with our result. The analyses and tests summarized above may indicate that the observed anticorrelation cannot be solely attributed to biases, but also to a true physical effect. We suggest that galaxy light concentration versus $\mathrm{X} / \mathrm{O}$ flux ratio relation traces the correlation between the nuclear BH mass and the Eddington ratio. This result could therefore indicate that more concentrated or bulge-dominated (early Hubble-type) galaxies, having poor matter supply to feed the activity, have lower Eddington rates than those of late-type, with larger reservoirs of the material for AGN feeding. This suggested approach is consistent with some of the previous results (e.g., Wu \& Liu 2004; Ballo et al. 2006,

\footnotetext{
18 http://subarutelescope.org/Science/SubaruProject/SDS/
}

2007; Kawakatu et al. 2007). For more details on the analysis of possible bias effects, comparison with galaxy mock catalogs, and discussion of the observed anticorrelation see P09.

\section{SUMMARY AND CONCLUSIONS}

We processed and analyzed public, $200 \mathrm{ks}$ Chandra/ACIS observations of three fields comprising the original GWS gathered from the Chandra Data Archive, combined with our OTELO broadband survey carried out with the $4.2 \mathrm{~m}$ WHT at La Palma. Our X-ray catalog contains 639 unique X-ray emitters. Of these, $429(67 \%)$ objects have been detected above the $3 \sigma$ significance level, having a limit flux of $4.8 \times 10^{-16} \mathrm{erg} \mathrm{cm}^{-2} \mathrm{~s}^{-1}$ and a median flux of $1.81 \times 10^{-15} \mathrm{erg} \mathrm{cm}^{-2} \mathrm{~s}^{-1}$ in the full $(0.5-7 \mathrm{keV})$ band. Cross-matching the X-ray catalog with our optical broadband catalog, we found 340 X-ray emitters with optical counterparts. The completeness and the reliability of cross-matching procedure are $97 \%$ and $88.9 \%$, respectively. At our flux limit, the number counts of our complete X-ray sample are in good agreement with Nandra et al. (2005) and other comparison surveys mentioned in Section 2.1.2.

We performed a morphological classification obtaining and analyzing different structural parameters, including the $\mathrm{B} / \mathrm{T}$, residual parameter, Sérsic index, asymmetry and concentration indexes. We also performed the classification based on visual inspection. For a sample where most of the objects are faint $(R \geqslant 22)$ and with small isophotal area ( $\leqslant 300$ pixels), bulge-tototal flux ratio has revealed inaccurate to perform the morphological classification. The concentration index combined with the asymmetry index have been found to provide the best separation criterion for such a sample. Different morphological types have been observed to distribute according to the following ranges of concentration index: group 0 (compact objects) $\rightarrow \mathrm{C} \geqslant 0.7$ (or CLASS_STAR $\geqslant 0.9$ ), group I (E, E/S0 and S0) $\rightarrow 0.45 \leqslant \mathrm{C}<0.7$, group II (S0/S0a-Sa) $\rightarrow 0.3 \leqslant \mathrm{C} \leqslant 0.45$ (0.47), group III (Sab-Scd) $\rightarrow 0.15 \leqslant \mathrm{C} \leqslant 0.3$, and group IV (Sdm-Irr) $\rightarrow \mathrm{C} \leqslant 0.15$. The electronic table summarizes the morphological classification of all $340 \mathrm{X}$-ray emitters with optical counterparts and measured structural parameters.

To obtain the X-ray classification, we applied a criteria based on the correlation between $\mathrm{X} / \mathrm{O}$ flux ratio and $\mathrm{HR} 1^{\prime}$ hardness ratio. $63 \%$ of our sources, with $\mathrm{HR} 1^{\prime} \neq 0$, have been classified as X-ray type $1 \mathrm{AGN}$. 
Table 3

X-ray Emitters with Optical Counterparts in GWS Field

\begin{tabular}{|c|c|c|c|c|c|c|c|c|c|}
\hline ID & $\begin{array}{l}\text { R.A. } \\
\text { (deg) }\end{array}$ & $\begin{array}{l}\text { Decl. } \\
(\mathrm{deg})\end{array}$ & $\begin{array}{c}\text { Flux } \\
\left(\operatorname{erg~cm}{ }^{-2} \mathrm{~s}^{-1}\right)\end{array}$ & $\mathrm{X} / \mathrm{O}$ & $\mathrm{HR}^{\prime}{ }^{\prime}$ & $\mathrm{C}$ & A & Morph & $\overline{\text { AEGISX }}$ \\
\hline 1 & 213.9250276 & 52.3228123 & $1.89 \mathrm{E}-14$ & 0.91 & -0.62 & 0.415 & & Compact & egs_0131 \\
\hline 2 & 213.9255963 & 52.368818 & $1.73 \mathrm{E}-14$ & 0.47 & 0.12 & 0.608 & 0.06 & Compact & egs_0132 \\
\hline 3 & 213.9270976 & 52.3182987 & $8.80 \mathrm{E}-16$ & & & 0.161 & 0.09 & III & egs_0133 \\
\hline 4 & 213.9374623 & 52.3690383 & $4.45 \mathrm{E}-15$ & 3.94 & 0.10 & 0.159 & 0.04 & $\mathrm{~N}_{\mathrm{I}}$ & egs_0137 \\
\hline 5 & 213.939902 & 52.2168915 & $4.58 \mathrm{E}-16$ & & & 0.142 & 0.06 & IV & \\
\hline
\end{tabular}

(This table is available in its entirety in a machine-readable form in the online journal. A portion is shown here for guidance regarding its form and content.)

We presented the first results on the two-point angular correlation function of the X-ray selected sources in the Groth field from Chandra/ACIS observations, segregated in four subsamples: soft, hard2, $\mathrm{HR}_{(2-4.5 \mathrm{keV} / 0.5-2 \mathrm{keV})}$, and optical extended counterparts. We obtain the following results and conclusions:

1. All subsamples reveal a significant positive clustering signal in the $\sim 0.5-6^{\prime}$ separation regime. Canonical power law $(\gamma=1.8)$ fits to the angular correlation functions have been made, obtaining correlation lengths between $\sim 7^{\prime \prime}$ and $27^{\prime \prime}$, depending on the subsample.

2. We have neither found substantial differences between the clustering of soft and hard 2 selected X-ray sources, nor when comparing the populations of X-ray type 1 and type 2 AGN (i.e., "unobscured" and "obscured" AGN, respectively), separated using the $\mathrm{HR}(2-4.5 \mathrm{keV} / 0.5-$ $2 \mathrm{keV}) \approx-0.35$ boundary.

3. The clustering analysis of the optical counterparts of our $\mathrm{X}$-ray emitters provides a correlation length significantly larger than the one found for the whole galaxy population with the same limiting magnitude, and same field, but similar to that of strongly clustered populations like red and very red (bulge-dominated) galaxies. A clustering signal $>1 \sigma$ was observed even at $\theta \sim 30^{\prime \prime}$, which corresponds to $160 h^{-1} \mathrm{kpc}$, assuming a mean redshift of 0.85 for Chandra extragalactic sources (Hasan 2007). This comoving distance is, for instance, comparable to the separation of two mergers in the $t \approx 2$ Gyr stage of their large-scale evolution, according to the model of Mayer et al. (2007) on rapid formation of super massive black hole. Hence, our results suggest that the environment plays an important (but not unique) role as possible triggering mechanism of AGN phenomena. This fact can be perfectly compatible with the interpretation given to the anticorrelation between $\mathrm{X} / \mathrm{O}$ ratio and the concentration index. The former may be associated to the genesis of the AGNs, while the anticorrelation would rather be related to their maintenance and evolution.

We have investigated the correlations between X-ray and broadband optical structural parameters that provide with information about the host galaxy morphology and populations, finding that:

1. No clear separation has been found between morphology and the X-ray type, but most of our objects classified as compact are placed in the region of X-ray type 1 AGNs.

2. We confirm a tendency of X-ray type 1 objects to show bluer colors when compared with X-ray type 2 sources.

3. Finally, we confirmed an anticorrelation between the $\mathrm{X}$-ray-to-optical flux ratio and the concentration index (which parameterizes the morphology), already studied in P09, with significance level higher than $99 \%$, showing that the early-type galaxies tend to have lower and the late-type galaxies higher than average $\mathrm{X} / \mathrm{O}$ values. Objects classified as compact show this anticorrelation as well, with the same significance level. The anticorrelation might suggest that early-type galaxies, having poor matter supply to feed the activity, have lower accretion rates than those of late type, with larger reserves of gas for AGN feeding.

We thank the anonymous referee for a number of valuable comments and suggestions which contributed to improve the quality of this paper. We would also like to thank Laura Tomás who kindly provided us with her software for diagnosis of hardness ratios using model grids. This work was supported by the Spanish Plan Nacional de Astronomía y Astrofísica under grants AYA2006-2358 and AYA2008-06311-C02-01. J.I.G.-S. and J.G. acknowledge financial support from the Spanish Ministerio de Educación y Ciencia under grants AYA2008-06311C02-02 and AYA2006-2358, respectively. This research has made use of software provided by the Chandra X-ray Center (CXC) in the application packages CIAO and ChIPS. IRAF is distributed by the National Optical Astronomy Observatory, which is operated by the Association of Universities for Research in Astronomy (AURA) under cooperative agreement with the National Science Foundation. This publication makes use of data products from the Two Micron All Sky Survey, which is a joint project of the University of Massachusetts and the Infrared Processing and Analysis Center California Institute of Technology, funded by the National Aeronautics and Space Administration and the National Science Foundation. We acknowledge support from the Faculty of the European Space Astronomy Centre (ESAC).

\section{APPENDIX}

\section{DESCRIPTION OF THE ELECTRONIC CATALOG}

The electronic catalog presents the data for the sample of 340 X-ray emitters with optical counterparts in GWS field analyzed in this paper. Table 3 shows an example of the format and content of the catalog. The column entries are as follows: (1) ID: Identifier number; (2 and 3) R.A., decl.: Equinox J2000.0 right ascension and declination. These coordinates correspond to the centroid in the broadband optical catalog see Section 2.3); (4) Flux: X-ray flux in the full $(0.5-7 \mathrm{keV})$ band; (5) $X / O$ : Xray-to-optical flux ratio, computed as the ratio of the observed $\mathrm{X}$-ray flux in the $0.5-4.5 \mathrm{keV}$ energy; (6) $H R l^{\prime}$ : (2-4.5/0.5$2 \mathrm{keV}$ ) hardness ratio computed as in Equation (1); (7) $C$ : Abraham concentration index, defined as the ratio between the integrated flux within certain radius defined by the normalized 
radius $\alpha=0.3$, and the total flux; (8) $A$ : Asymmetry index (see Section 3.1); (9) Morph: Final morphological classification. The groups from I to IV correspond to the ones defined in the Section 3.2. Objects assigned as compact are all objects with SExtractor CLASS_STAR parameter $\geqslant 0.9$. PM represents the possible mergers, while the objects assigned with $\mathrm{N}_{\mathrm{I}}$ are not identified; (10) AEGISX: Identifier in the AEGIS X-ray catalog Laird et al. 2009).

\section{REFERENCES}

Abraham, R. G., Valdes, F., Yee, H. K. C., \& van der Bergh, S. 1994, ApJ, 432 90

Abraham, R. G., van der Bergh, S., Glazebrook, K., Ellis, R. S., Santiago, B. X., \& Surma, P. 1996, ApJ, 107, 1

Alexander, D. M., Brandt, W. N., Hornschemeier, A. E., Garmire, G. P., Schneider, D. P., Bauer, F. E., \& Griffiths, R. E. 2001, AJ, 122, 2156

Alexander, D. M., et al. 2003, AJ, 126, 539

Ballo, L., et al. 2006, in Proc. X-ray Universe, Black Hole Masses and Eddington Ratios of AGNs Contributing Most of the XRB, ed. A. Wilson (ESA SP-604; Noordwijk: ESA), 595

Ballo, L., et al. 2007, ApJ, 667, 97

Barcons, X., et al. 2007, A\&A, 476, 1191

Barger, A. J., et al. 2003, AJ, 126, 632

Basilakos, S., Georgakakis, A., Plionis, M., \& Georgantopoulos, I. 2004, ApJ, 607, L79

Basilakos, S., Plionis, M., Georgakakis, A., \& Georgantopoulos, I. 2005, MNRAS, 356, 183

Bauer, F. E., et al. 2004, AJ, 128, 2048

Bertin, E., \& Arnouts, S. 1996, A\&AS, 117, 393

Boylan-Kolchin, M., et al. 2009, MNRAS, 398, 1150

Brandt, W. N., \& Hasinger, G. 2005, ARA\&A, 43, 827

Briggs, I. 1974, Geophysics, 39, 39

Bundy, K., et al. 2008, ApJ, 681, 931

Caccianiga, A., et al. 2004, A\&A, 416, 901

Capak, P., et al. 2007, ApJS, 172, 99

Cappelluti, N., et al. 2007, ApJS, 172, 341

Carrera, F. J., et al. 2007, A\&A, 469, 27

Cepa, J., et al. 2005, RevMexAA, 24, 82

Cepa, J., et al. 2008, A\&A, 490, 1

Civano, F., et al. 2007, A\&A, 476, 1223

Coil, A. L., Newman, J. A., Kaiser, N., Davis, M., Ma, Ch., Kocevski, D. D., \& Koo, D. C. 2004, ApJ, 617, 765

Comastri, A., et al. 2002, ApJ, 571, 771

Della Ceca, R., et al. 2004, A\&A, 428, 383

de Ruiter, H. R., Willis, A. G., \& Arp, H. C. 1977, A\&AS, 28, 211

Dickinson, M., et al. 2004, ApJ, 99, 122

Dwelly, T., et al. 2005, MNRAS, 360, 1426

Ferrarese, L., \& Merritt, D. 2000, ApJ, 539, L9

Fiore, F., Elvis, M., Maiolino, R., Nicastro, F., Siemiginowska, A., Stratta, G., \& D'Elia, V. 2003, A\&A, 409, 57

Fiore, F., et al. 2001, in AIP Conf. Proc., Vol. 599, X-ray Astronomy: Stellar Endpoints, AGN, and the Diffuse X-ray Background, ed. E. W. Nicholas, M. Giuseppe, \& G. C. P. Giorgio (Melville, NY: AIP), 111

Furusawa, H., et al. 2008, ApJS, 176, 1

Gabor, J. M., et al. 2009, ApJ, 691, 705

Gandhi, P., et al. 2006, A\&A, 457, 393

Gebhardt, K., et al. 2000, AJ, 119, 1157
Georgakakis, A., et al. 2006, MNRAS, 371, 221

Georgakakis, A., et al. 2007, ApJ, 660, L15

Giacconi, R., et al. 2002, ApJS, 139, 369

Gilli, R., et al. 2003, ApJ, 592, 721

Gilli, R., et al. 2005, A\&A, 430, 811

Graham, A., Erwin, P., Caon, N., \& Trujillo, I. 2001b, ApJ, 563, L11

Grogin, N. A., et al. 2003, ApJ, 595, 685

Hasan, P. 2007, Ap\&SS, 312, 63

Hasinger, G. 2008, A\&A, 490, 905

Im, M., et al. 2002, ApJ, 571, 171

Kauffmann, G., et al. 2003, MNRAS, 346, 1055

Kawakatu, N., et al. 2007, ApJ, 661, 660

Laird, E. S., et al. 2009, ApJS, 180, 102

Landy, S. D., \& Szalay, A. S. 1993, ApJ, 412, 64

Lilly, S. J., et al. 2007, ApJS, 172, 70L

Mainieri, V., et al. 2002, A\&A, 393, 425

Manners, J. C., et al. 2003, MNRAS, 343, 293

Mayer, L., Kazantzidis, S., Madau, P., Colpi, M., Quinn, T., \& Wadsley, J. 2007, Science, 316, 1874

Miyaji, T., et al. 2007, ApJS, 172, 396

Moretti, A., et al. 2003, ApJ, 588, 696

Mukai, K. 1993, Legac, 3, 21

Mushotzky, R. 2004, Supermassive Black Holes in the Distant Universe, 308, 53

Nandra, K., et al. 2005, MNRAS, 356, 568

Peebles, P. J. E. 1980, The Large Scale Structure of the Universe (Princeton, NJ: Princeton Univ. Press)

Perola, G. C., et al. 2004, A\&A, 421, 491

Pierce, C. M., et al. 2007, ApJ, 660, L19

Pović, M., et al. 2009, ApJ, 702, L51

Puccetti, S., et al. 2006, A\&A, 457, 501

Sánchez, S. F., et al. 2004, ApJ, 614, 586

Sánchez-Portal, M., Díaz, A. I., Terlevich, E., \& Terlevich, R. 2004, MNRAS, 350, 1087

Sánchez-Portal, M., Pérez García, A. M., Cepa, J., González-Serrano, J. I., Alfaro, E. J., Castañeda, H. O., Gallego, J., \& González, J. J. 2007, RevMexAA, 29, 175

Schade, D., Lilly, S. J., Crampton, D., Hammer, F., Le Fevre, O., \& Tresse, L. 1995, ApJ, 451, 1

Schade, D., Lilly, S. J., Le Fevre, O., Hammer, F., \& Crampton, D. 1996, ApJ, 464, 79S

Scoville, N., et al. 2007, ApJS, 172, 1S

Silverman, J. D., et al. 2008, ApJ, 675, 1025

Simard, L. 1998, in ASP Conf. Ser. 145, GIM2D: An IRAF Package for the Quantitative Morphology Analysis of Distant Galaxies, ed. R. Albrecht, R. N. Hook, \& H. A. Bushouse (San Francisco, CA: ASP), 108

Simard, L., et al. 2002, ApJS, 142, 1S

Steffen, A. T., Strateva, I., Brandt, N., Alexander, D. M., Koekemoer, A. M., Lehmer, B. D., Schneider, D. P., \& Vignali, C. 2006, AJ, 131, 2826

Stetson, P. B. 1987, PASP, 99, 191

Taylor, M. B. 2005, in ASP Conf. Ser. 347, TOPCAT \& STIL: Starlink Table/ VO Table Processing Software, ed. P. Shopbell, M. Britton, \& R. Ebert (San Francisco, CA: ASP), 29

Ueda, Y., et al. 2008, ApJS, 179, 124

Waskett, T. J., Eales, S. A., Gear, W. K., McCracken, H. J., Lilly, S., \& Brodwin, M. 2005, MNRAS, 363, 801

Worsley, M. A., et al. 2004, MNRAS, 352, L28

Wu, X., \& Liu, F. K. 2004, ApJ, 614, 91

Yang, Y., Mushotzky, R. F., Barger, A. J., \& Cowie, L. L. 2006, ApJ, 645, 68

Yang, Y., Mushotzky, R. F., Barger, A. J., Cowie, L. L., Sanders, D. B., \& Steffen, A. T. 2003, ApJ, 585, L85

Yee, H. K. 1983, ApJ, 272, 473 